\title{
Connecting polymer synthesis and chemical recycling on a chain-by-chain basis: a unified matrix-based kinetic Monte Carlo strategy
}

\author{
Kyann De Smit, ${ }^{a}$ Yoshi W. Marien, ${ }^{a}$ Kevin M. Van Geem, ${ }^{a}$ Paul H.M. Van Steenberge, ${ }^{a}$ Dagmar R. D’hooge ${ }^{\mathrm{a}, \mathrm{b}, *}$
}

One of the challenges faced by modern society is the realization of a circular economy for polymer products. A bottleneck is the understanding of (co)polymer synthesis and degradation routes on a chain-by-chain basis, as the location of specific functional groups or structural defects determines the distributed chemical nature of the macrospecies involved and thus the reaction possibilities and macroscopic properties. Here we present a unified matrix-based elementary step driven kinetic Monte Carlo (kMC) strategy to fully connect polymer synthesis and subsequent degradation at the molecular level, aiming at the recovery of the original monomer or a product spectrum of oligomers either degradable or upcyclable to high-added value products. This kMC strategy is illustrated for radical polymerization with methyl methacrylate (MMA) as the main monomer, selecting two case studies: (i) radical polymerization of MMA and the subsequent thermal degradation back to this monomer; and (ii) radical copolymerization of MMA with 2-methylene-1,3-dioxepane (MDO) and the subsequent hydrolysis of the resulting poly(MMA-MDO) toward biodegradable oligomers. For the first case study, it is shown that the shape and location of the log-molar mass distribution strongly affects the degradation efficiency. For the second case study, it is highlighted that the inherent molecular heterogeneity of copolymers strongly defines the framework in which degradation synthesis routes can be exploited.

Keywords: polymerization kinetics, depolymerization kinetics, macromolecular design, stochastic modeling, (bio)degradability

\section{Introduction}

Free radical polymerization (FRP) of vinyl monomers has been a cornerstone of the polymer industry during the last century, mainly thanks to its robustness to oxygen and impurities, and its short reaction times, which range from minute to hour scale. ${ }^{1,2}$ Recently, due to the growing environmental awareness, industry has shown interest in recycling FRP products. ${ }^{3,4}$ Examples of recyclable products are poly(methyl methacrylate) (PMMA) materials, which are often used as glass substitutes (e.g. face protection) due to their transparency and excellent optical properties. ${ }^{5,6}$ Thermochemical recycling of PMMA at elevated temperature is specifically interesting because of the high monomer recovery (>90\%), which is quite unique for vinyl polymers. ${ }^{7}$ The reason is that PMMA degrades mainly through fast consecutive radically induced depropagation reactions or so-called unzipping, ${ }^{8-10}$ which produces ideally MMA at the end of each unzipping cycle. The radicals are formed by an initiation mechanism usually involving specific structural defects formed during the PMMA synthesis. Examples are head-to-head defects and unsaturated chain ends following from termination by recombination and disproportionation respectively. ${ }^{11-15}$ The head-to-head defects alternatively follow from less common head-to-head additions.

One can imagine that the location and concentrations of these structural defects, in general functional groups, has an immense effect on the thermal PMMA degradation kinetics, as a certain thermal energy is only capable to break/alter certain chemical bonds/moieties. The exact thermal degradation reaction mechanism - even for virgin PMMA - is still under debate. ${ }^{6}$ Most researchers adopt the multi-step degradation mechanism originally proposed by Kashiwagi et al. ${ }^{11,12,16}$ because it is the most straightforward mechanism to predict thermogravimetric analysis (TGA) measurements, in which mass losses are recorded for a given heating rate. With increasing temperature it is claimed that one observes unzipping by initiation through head-to-head fission (see red star in Figure 1), unsaturated end-chain fission, and head-to-tail fission, as supported by many TGA results displaying three dominant peaks and thus three cycles of mass losses. ${ }^{17,18} \mathrm{~A}$ fine tuning as proposed by Manring ${ }^{13-15,19}$ considers the interplay of chemical reactions and molecular diffusion, favoring degradation reactions leading to small radicals which are inherently more mobile. ${ }^{20,21}$ 


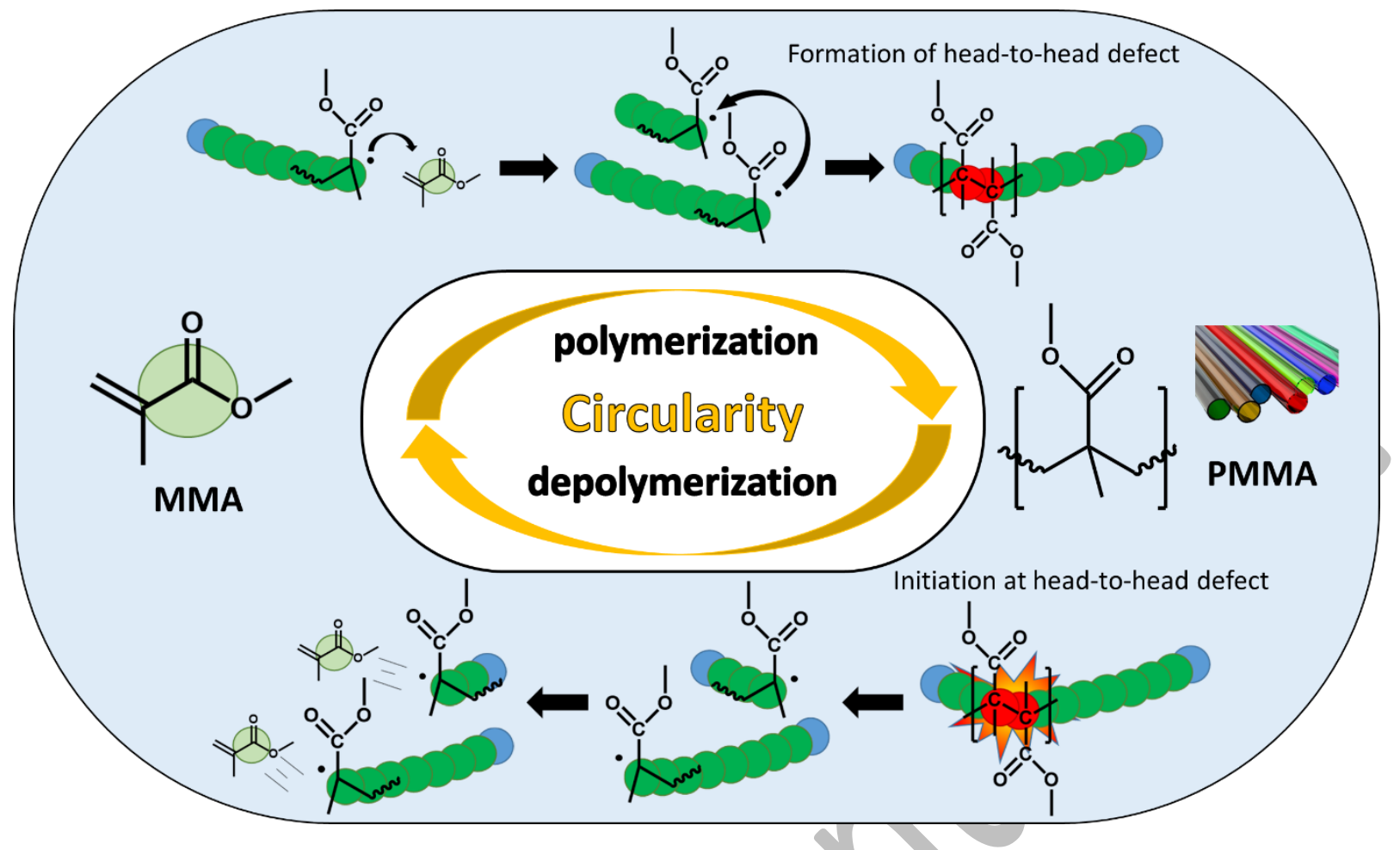

Figure 1: Circularity for vinyl polymers with the upper branch free radical polymerization (FRP) and the lower branch the thermochemical recycling or thermal degradation aiming at monomer recovery. Focus is on linear polymer poly(methyl methacrylate (PMMA). The spheres indicated in red are examples of a structural defect at which degradation can be initiated; only termination by recombination is shown

Manring also put forward that radicals of a general source (e.g. through a hydrogen abstraction based on an impurity) can add to unsaturated end-chain ends, leading to an alternative initiation mechanism. This need of extra initiation mechanisms to unravel PMMA thermal degradation kinetics follows from the observation that sometimes four instead of three peaks are witnessed in TGA analysis. ${ }^{6,8,22,23}$

A challenge is thus the linkage of PMMA synthesis by FRP on the elementary reaction level to the follow-up thermochemical recycling still at this elementary reaction level in view of the implementation of circularity for polyacrylics, as conceptually highlighted in Figure 1. Essential is the consideration of the dominant reaction pathways depending on the specific experimental synthesis and thermochemical recycling conditions.

This direction toward chemically oriented research for chemical recycling goes hand in hand with the development of new (bio)degradable polymers as well. A challenge here is to incorporate biodegradable groups in vinyl polymer synthesis, benefiting from the progress in the field of biopolymers, more specifically the synthesis of aliphatic polyesters such as polycaprolactone (PCL), polylactide (PLA) and polyglycolide (PGA) as typically synthesized by ring opening polymerization (ROP). ${ }^{24,25}$ The major drawback of these polymers is that their application scope is rather limited and that ROP generally demands more stringent synthetic conditions with respect to pressure and temperature. ${ }^{25,26}$ Hence, recent attempts in the polymer chemistry community have combined the best of both worlds, i.e. the development of vinyl pseudo-homopolymers with (bio)degradable ester linkages has been explored through radical ROP of vinyl monomers with cyclic ketene acetal monomers (CKA's). ${ }^{26,27}$

In the context of next-generation PMMA synthesis one can put forward the polymerization of MMA and 2-methylene-1,3dioxepane (MDO). The (bio)degradation proceeds in two distinct stages, with in the first stage hydrolysis of the ester groups resulting in vinyl oligomer formation and in the second stage the biodegradation of these oligomers by bacteria. ${ }^{27,28} \mathrm{As}$ for the conventional thermochemical recycling it is essential to know the location of the degradable functional groups in the vinyl-like chains as they are triggering the degradation. Recent work of Gigmes et al. ${ }^{26}$ already highlighted the need of suited reactivity ratios under batch conditions. Once a CKA unit is incorporated cross-propagation should be taking place with consequently several vinyl monomer unit incorporations to enable the pseudo-homopolymer character. Here one aims at a controlled vinyl monomer segment length distribution (SLD) in view of potential classification as a (bio)degradable polymer.

Hence, it is essential to have detailed molecular information on a chain-by-chain basis both for thermochemical recycling of conventional PMMA (waste) and the synthesis of next-generation PMMA with (bio)degradable units. Such knowledge would allow 
for each chain at any reaction time - either for the polymer synthesis or thermochemical recycling - to verify what the individual locations of all (co)monomer unit types and functional groups are. To acquire that level of structural detail

kinetic modeling is a powerful tool as it can access the concentration evolutions of many types of molecules and thus an evaluation of product spectra along the progress of the chemical modification considered. ${ }^{8,14}$ Despite that the modeling of FRP has been a research field for decades ${ }^{29,30}$ a detailed chain-by-chain kinetic modeling is rather scarce as traditionally in both the experimental and modeling community no direct link exists to a subsequent (thermo)chemical recycling. For most FRP applications (e.g. packaging materials and household goods) knowledge about basic/overall polymer properties such as the mass average molar mass, dispersity and branching density usually suffices to categorize the polymer as eligible. Only for specialty polymers, such as block/gradient copolymers and coating precursors as made for instance by more dedicated reversible deactivation radical polymerization techniques, it already became clear that optimal synthesis conditions require more advanced modeling tools. ${ }^{31-36}$

Furthermore, the older kinetic models typically rely on more basic deterministic techniques ${ }^{37,38}$ aiming understandably only at the prediction of the monomer conversion and average molecular properties, specifically average chain lengths. ${ }^{39}$ However, even "simple" polymers such as PMMA $^{39-41}$ are distributed with respect to chain length, while the synthesis is characterized by side reactions and chain length dependent reactivities caused by a interplay of chemistry and diffusional limitations. 42,43 Hence, one needs to solve a very high amount of strongly coupled differential equations to deterministically describe the kinetics of populations of macromolecule types. ${ }^{44}$ At one point numerical issues are unavoidably encountered and only a limited number of specialized deterministic techniques have been developed to tackle distribution effects, typically for more basic reaction schemes and intrinsic kinetics. ${ }^{45-48}$

The mathematical treatment of several population types in radical polymerization is more elegantly tackled by kinetic Monte Carlo $(k M C)$ techniques, which have become very popular in the recent decades due to the increased computational power and the ease of implementation. ${ }^{20,49-52}$ The basis of $k \mathrm{MC}$ techniques is the stochastic simulation algorithm (SSA) as proposed by Gillespie et al. ${ }^{53}$ Starting from a sufficiently high amount of initial molecules, SSA randomly samples reaction event by reaction event in a discrete manner in accordance with the reaction probabilities that are dictated by so-called MC or microscopic reaction rates as expressed in frequencies thus $\mathrm{s}^{-1}$. In radical polymerization, many reaction events can take place and specific focus has been on the development of $k \mathrm{MC}$ algorithms in which chain length dependencies either on the reaction (variation in reactivities) or molecule (variation in concentrations) level are efficiently addressed. ${ }^{20,48,54}$ Originally the focus was on the use of binary tree structures but to enable detailed molecular information on a chain-by-chain basis matrix-based $k M C$ algorithms are recommended. ${ }^{30,55,56}$ Every cell in matrix format allows to store specific information regarding the local composition of an individual chain, e.g. its monomer sequences. Most studies have however dealt with low average chain length polymerization processes and simplified reaction schemes with a limited amount of structural parameters or defects. ${ }^{11,12,14,15,19,57,58}$ For instance, in these $k M C$ models, the main objective is only on the tracking of end-chain unsaturations but no differentiation is made based on addition steps thus not allowing a mapping of head-tail, head-head, tail-head and tail-tail structural parameters along the chains, as required to fully address the circularity aspect from Figure 1. In most FRP kinetic studies one only considers head-to-tail propagation although some exceptions exist specifically for vinyl acetate as monomer. ${ }^{59-61}$

To lift the applicability of matrix-based kMC techniques to the field of (i) commercial thermochemical recycling thus involving high average chain length polymers and (ii) next-generation polymer synthesis of vinyl polymers a further design of the underlying implementation principles is needed, as currently limitations regarding computer capacity and appreciation in simulation time are manifested. In the past some Monte Carlo techniques have been developed for modeling the degradation of polymeric materials. Some efforts include lattice based models which represent the polymeric material in a 2/3D matrix, where each node corresponds to a piece of the 3D polymer material. ${ }^{62-66} \mathrm{~A}$ recent study by Adema et al. ${ }^{66}$ discusses the photodegradation of polyester-urethane coatings, where the coatings are subdivided into small cubes. These cubes are an ensemble of different chemical moieties and the degradation of some of these moieties is modeled using an elementary reaction scheme. Other efforts include the modeling of the thermal degradation of polymeric materials specifically. ${ }^{67-75}$ Most of them however, are not elementary reaction step driven as they generally adopt a very basic reaction scheme (e.g. only random chain fission) with mostly first order reactions and involve TGA analyses with overall degradation rates. Some studies have modeled the thermal degradation of polymers in a very detailed way making use of a complex and extensive reaction network 67,68 , however focus only on the degradation and the input for the degradation model is generally simulated based on theoretical distributions or experimentally determined SEC-traces. Also, general Monte Carlo models have been used to study the time evolution of the polymer molecular weight distribution during degradation in linear ${ }^{71}$, cross-linked ${ }^{76}$, branched ${ }^{77-79}$ and star-shaped polymers ${ }^{80}$. These studies involve random sampling and chain fission probabilities to study the degradation of polymer chains, but the fission probabilities are not related to rate coefficients of the elementary reaction steps. In this work, a novel matrix-based elementary reaction step driven isothermal lab-scale kMC modeling algorithm is therefore presented, which keeps track of individual molecular structures of chains both during radical polymerization and subsequent thermochemical recycling or degradation, whilst using a novel compression method for the storage of long polymer chains. 
First the potential of the algorithm is demonstrated for PMMA circularity, considering a typical lab-scale bulk isothermal FRP of MMA involving initiation, chain initiation, propagation, termination and chain transfer to monomer as key elementary reactions, and for the subsequent isothermal thermochemical recycling the dominantly considered degradation mechanism as

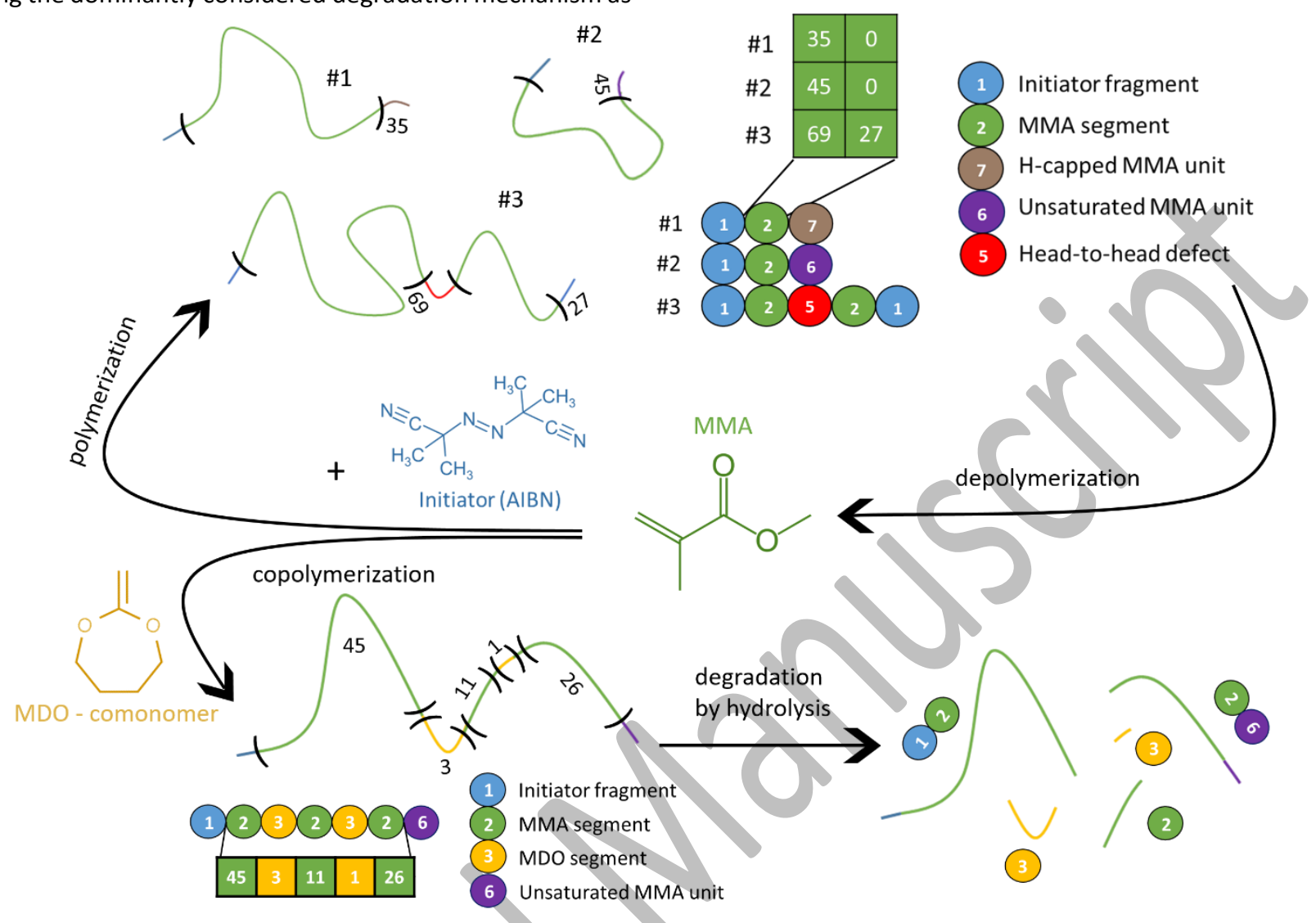

Figure 2: Concept of main matrix (spheres) and submatrix (squares) to visualize the detailed molecular structure per chain at a given time either during synthesis or thermochemical recycling/degradation; top part: focus on test case 1; bottom part focus on test case 2 . The matrices are updated in the actual kinetic Monte Carlo algorithm as included in Figure S2

introduced by Kashiwagi et al. ${ }^{12}$ Corrections for diffusional limitations are considered via apparent rate coefficients that are determined based on fundamental theories as developed before. ${ }^{81-84}$ Moreover, the general applicability of the developed compression-based algorithm is further highlighted by also including a chain-by-chain mapping for the synthesis and degradation of a PMMA-like polymer with MDO degradable units. For both test cases, specific focus is on the temporal variation of the logmolar mass distribution (MMD), starting from an accurately generated initial MMD trace as predicted by the radical polymeriza tion kinetic model.

\section{Principles and parameters of kinetic Monte Carlo model}

In this section, we first highlight the principles of the developed matrix-based $k \mathrm{MC}$ model to fully connect the chemistry during polymer synthesis and subsequent thermochemical recycling or degradation. Then, the essential model parameters are discussed, focusing on two lab-scale single phase isothermal test cases: (i) FRP of MMA with 2,2'-dimethyl-2,2'-azodipropiononitril (AIBN) as conventional radical initiator ${ }^{84}$ and the subsequent thermochemical recycling of this FRP made PMMA (test case 1 ) and (ii) radical copolymerization of MMA and MDO, again considering AIBN, and the subsequent degradation by hydrolysis under lab-accelerated conditions $^{26}$ (test case 2 ).

\section{Matrix-based kMC principles}

Starting from our matrix-based $k M C$ platform to track individual monomer sequences in polymerizations aiming for short average chain lengths (e.g. $<500)^{30,56,85}$ we introduce here new matrix-based concepts to elegantly model in a first phase conventional/free, thus high average chain length, radical homopolymerization of a vinyl monomer (e.g. MMA) and also related relevant copolymerizations involving e.g. MDO or more conventional comonomers such as acrylates. The $k \mathrm{MC}$ model tracks the detailed molecular structure of a sufficiently large number of individual chains to enable numerical convergence, $86-88$ including not 
only monomer sequences but also structural parameters (e.g. the location of specific side groups and functionalities) and defects (e.g. head-to-head) as a function reaction time.

Based on this detailed explicit molecular information traditional average characteristics such as the number/mass average chain length $\left(x_{n} / \mathrm{m}\right)$ are accurately calculated in parallel. The explicit visualization at the level of the individual macrospecies is crucial input for the correct representation of the thermochemical degradation kinetics as well. As explained above, the initiation

Table 1: Reactions, Arrhenius parameters and (intrinsic) rate coefficients for free radical polymerization (FRP) of methyl methacrylate (MMA) with 2,2'-dimethyl-2,2'azodipropiononitril (AIBN); Figure S3 in the Supporting Information depicts the chemical changes.

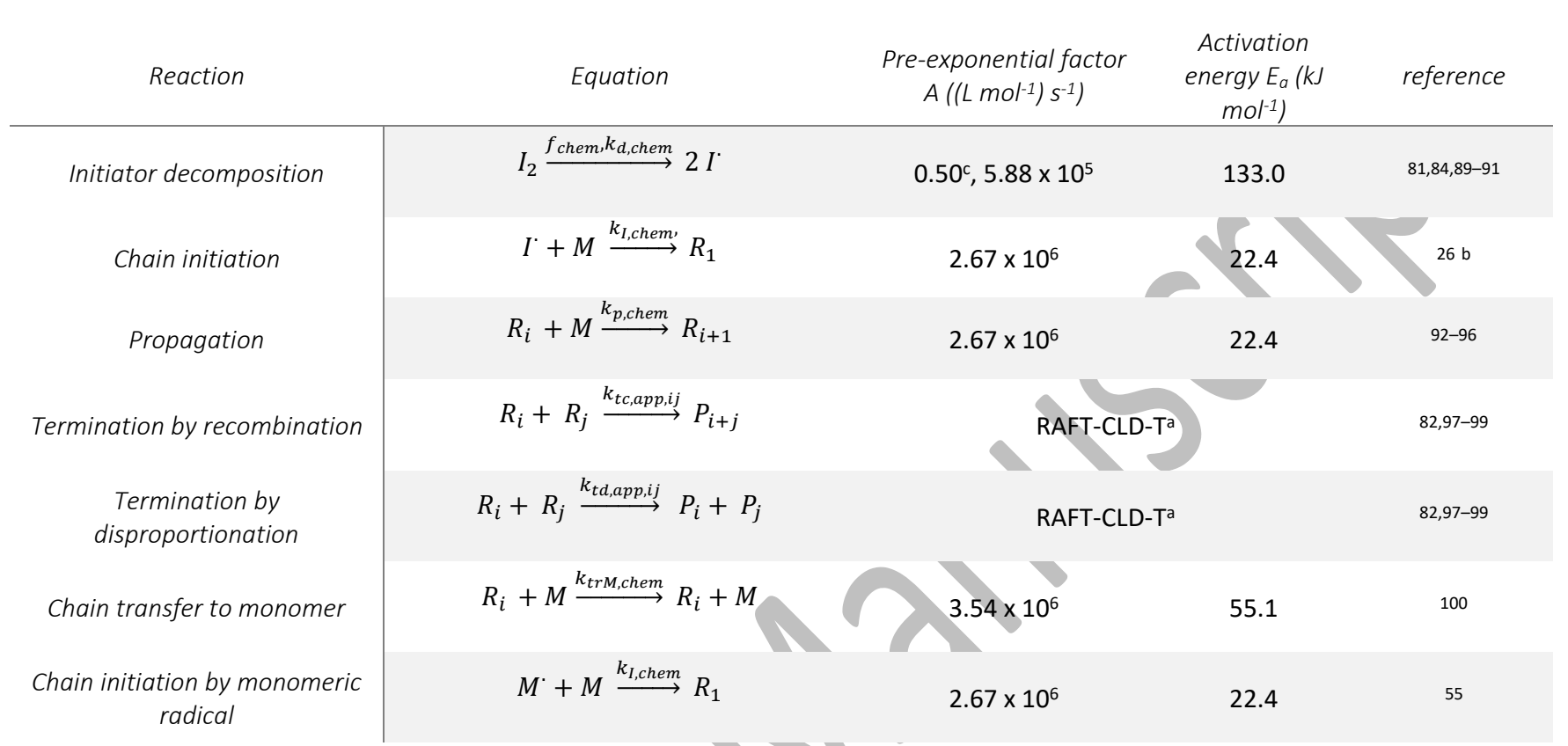

a Parameters of reversible addition fragmentation chain transfer - chain length dependent - termination (RAFT-CLD-T) model in Table 2, with the corresponding equations in the Supporting Information; for other reactions also the consideration of apparent reactivities ( $k_{\text {app }}$ values and $f_{\text {app }}$ value), corresponding equations in Supporting Information; ${ }^{b}$ based on this reference the same reactivity as for the conventional chain initiation is considered; ${ }^{c}$ dimensionless and initial value from model of Buback et al..$^{84}$

of a thermal degradation mechanism usually starts at certain structural defects/functionalities in the polymer backbone. Hence, the ideal input for the simulation of the thermal degradation chemistry is explicit information regarding the presence and location of specific functional groups and defects responsible for a certain degradation mechanism.

As visible in Figure 2 (top part) focusing for simplicity only on 3 chains at a given moment present in FRP of MMA or the subsequent thermal degradation, this detailed molecular information is stored by using a main matrix and a submatrix in contrast to our previous work on $k \mathrm{MC}$ modeling regarding the synthesis of linear (or slightly branched) polymers in which only one matrix type has been used. The main matrix is a sequence matrix with its labels (spheres in Figure 2: top part) highlighting the nature of the sequence of chemical moieties/structural defects present in the selected chain from "left" to "right". A label can refer for instance to an initiator fragment, a monomer sequence (thus a series of MMA units), a head-to-head defect, and a (un)saturated chain end. The submatrix (squares in Figure 2: top part) provides for the consecutive labels of monomer sequences in the main matrix the actual lengths of the monomer sequences, explaining the name segment length matrix. The concept of a main and a submatrix is further highlighted at the bottom part of Figure 2, focusing on one chain in the second test case, the radical copolymerization of MMA and MDO. The consecutive numbers in the segment length matrix automatically reflect a switch of the comonomer type from MMA to MDO or vice versa.

The basis of this elementary reaction step driven model is Gillespie's algorithm, ${ }^{53}$ which is explained in detail in the supporting information (see Figure S1 and S2). To facilitate the identification of certain matrix positions and to store molecular information upon executing the $k \mathrm{MC}$ algorithm efficient data structure rules are required, as explained in more detail in the Supporting Information (cf. Figure S2). Specifically the separation in a main and submatrix allows for compression of the former while in any case keeping the correct chain length, even for the longest chains present by elegantly utilizing the last element of each row. This implies that the tail of the MMD is accurately represented, which solves a challenge in the field of polymerization kinetics with typically unavoidable extensive noise at the higher chain length region. ${ }^{101}$ In the present work such noise remains only relevant for the very extreme chain lengths representing species with very low concentrations. At these extreme chain lengths an averaging 
based on a spline correction is considered. Upon accounting for size exclusion chromatography (SEC) broadening one can additionally compare with measured SEC data but in the present work such broadening factor is not included, as no direct comparison with actual experimental distributions is considered. For benchmarking more attention is given to the related $\mathrm{MMD}$ averages for which broadening is less an issue, at least to a first approximation. ${ }^{102}$

\section{Reactions and kinetic parameters for test case 1: phase 1: FRP of MMA}

Table 1 (column 1) shows the reactions for bulk FRP of MMA with AIBN as conventional radical initiator. Basic FRP reactions such as conventional radical initiation, (head-to-tail) propagation and termination by recombination and termination are included, complemented with side reactions such as chain

Table 2: Reversible addition fragmentation - chain length dependent - termination (RAFT-CLD-T) parameters as required in Table 1 to calculate chain length and monomer conversion $\left(X_{m}\right)$ dependent apparent termination rate coefficients for bulk free radical polymerization (FRP) of methyl methacrylate (MMA; equations: Supporting Information). The fraction of termination by disproportionation is taken equal to 0.73 and based on the work of Nakamura et al. ${ }^{103}$

\begin{tabular}{|c|c|c|c|c|c|c|}
\hline Temperature & $i_{g e l}$ & $i_{S L}$ & $\alpha_{s}$ & $\alpha_{l}$ & $\alpha_{g e l}$ & $\log \left(\boldsymbol{k}_{t, 11, a p p}\right)$ \\
\hline$>323 K^{82,97}$ & $0.53 X_{m}^{-2.5}$ & 100 & 0.65 & 0.15 & $1.66 X_{m}-0.06$ & 9.1 \\
\hline$\leqq 323 K^{a}$ & $3.12 \mathrm{X}_{\mathrm{m}}^{-1,8}$ & 100 & 0.65 & 0.15 & $2.20 x_{m}-0.06$ & 9.1 \\
\hline
\end{tabular}

a this work

transfer to monomer and subsequent chain initiation by the monomeric radical fragment. The last reaction leads to a chain end that resembles that of a dead chain formed through termination by disproportionation but needs to be stored separately from a kinetic point of view (other colored sphere in Figure 2: top part). The competition between termination by disproportionation and recombination is important to characterize the PMMA at the inlet of a subsequent thermochemical recycling process. In particular, the location and amount of termination defects is important to account for various degradation initiation mechanisms. In the present work, the relative importance of both termination modes is based on the work of Nakamura et al. ${ }^{103}$

Also the corresponding Arrhenius parameters to calculate chemical thus intrinsic rate coefficients ( $k_{\text {chem }}$ values) are provided in Table 1. These coefficients are all based on literature data. ${ }^{26,55,81,61,68-100}$ For termination apparent rate coefficients ( $k_{t, \text { app }}$ values) are however directly used. For such bimolecular reactions, even for low monomer conversions, diffusional limitations are already relevant, ${ }^{20,81,104}$ since the mobility of two (macro)radicals in a viscous environment is easily restricted and strongly chain length dependent. This is the so-called gel- or Trommsdorff effect ${ }^{105}$ so that the average apparent termination reactivities $\left(<k_{t(c / d), \text { app }}>\right)$ drop, leading to a rate acceleration and increase in average chain length at one point in the FRP. Depending on the nature of the reaction mixture and monomer type, this switch is truly manifesting between $X_{m}$ equal to 20 and $70 \%{ }^{2}$

In the last decades, extensive efforts have been made to develop fundamental methods to predict $k_{\mathrm{t}(\mathrm{c} / \mathrm{d}) \text {,app }}$ as a function of both chain length and polymer mass fraction or $X_{\mathrm{m}}{ }^{21,54,97,106}$ One of the most promising methods is the composite $k_{\mathrm{t}}$ model, which is also known as the reversible addition fragmentation - chain length dependent - termination (RAFT-CLD-T) model of which the key equations are mentioned in Section S2 of the Supporting Information. The corresponding parameters for polymerization temperatures above $323 \mathrm{~K}$ are directly taken from the work of Johnston-Hall and Monteiro ${ }^{82,97}$ and are listed in Table 2 . At lower temperatures preliminary screening in this work revealed that part of these parameters needed to be adapted, which is not surprising as such temperatures are further away from the glass transition temperature of the polymer so that less mobility is expected. The reported changes are within expected variations with specifically an activation energy for termination between macroradicals of unity chain length equal to $5.6 \mathrm{~mol}^{-1} .93,94$

Also for the other reactions in Table 1 we need to account for diffusional limitations. Apart from the gel-effect, there are two other main phenomena that relate to diffusional limitations in FRP: the glass and cage effect. The former relates to a decrease in the apparent (to a first approximation chain length independent) propagation rate coefficient $k_{\mathrm{p}, \text { app }}$ at high $X_{\mathrm{m}}$ at temperatures typically below the glass transition temperature of the reaction mixture. ${ }^{104,107}$ The latter describes the decrease in the apparent initiator efficiency $f_{\text {app }}$ with increasing $X_{\mathrm{m}}$. In the present work free volume based theories are considered based on the work of Buback et al. ${ }^{84}$ The relevant equations and literature-based parameters ${ }^{48,81}$ are given in Section S2 of the Supporting Information. To describe the glass effect over a broad temperature range (323-363 K) the current work revealed an optimized monomer diffusion activation energy of $23.5 \mathrm{~kJ} \mathrm{~mol}^{-1}$ to accurately grasp the kinetics of the lower temperature range (323 K) compared to value of $12 \mathrm{~kJ} \mathrm{~mol}^{-1}$ for the higher temperature range $(>323 \mathrm{~K}$ ). 


\section{Reactions and kinetic parameters for test case 1: phase 2: the degradation of FRP made PMMA}

In strong contrast to the well-accepted relevant elementary reactions and rather accurately known associated intrinsic and diffusion parameters in FRP of MMA is the almost absent kinetic investigation of the thermochemical recycling of PMMA at the elementary reaction level. ${ }^{6}$ This statement can be even generalized for thermochemical recycling of other vinyl polymers with the most fundamentally oriented results obtained in the Broadbelt research group regarding polystyrene thermal degradation. ${ }^{57,58}$

It is therefore not surprising that almost no elementary degradation kinetic parameters are available for PMMA thermochemical recycling. Focus has been on global kinetic models in which a full degradation mechanism, as in reality occurring via elementary reaction steps, is formally described as a single "reaction" based on peaks in mass losses during TGA with predetermined temperature programs. With such models

Table 3: Kinetic parameters at $623 \mathrm{~K}$ as used in combination with the reaction scheme in Figure 3 to describe the thermal degradation chemistry of poly(methyl methacrylate) (PMMA) made by free radical polymerization. For simplicity radicals are described by an average reactivity explaining the presence of $\mathrm{R}$ symbols at the product side with differentiation based on chain length only; M: MMA; for head-to-tail fission rate coefficient per monomer unit.

\begin{tabular}{|c|c|c|c|}
\hline reaction & equation & Rate coefficient $k$ & dimension \\
\hline $\begin{array}{c}\text { Initiation by head-to-head fission in } \\
\text { saturated chain }\end{array}$ & $P_{H-H, i+j} \stackrel{k_{I, H-H, \text { chem }}}{\longrightarrow} R_{i}+R_{j}$ & $1.5 \times 10^{-2}$ & $s^{-1}$ \\
\hline $\begin{array}{l}\text { Initiation by fission at unsaturated chain } \\
\text { end }\end{array}$ & $P_{=, i} \stackrel{k_{I,=, \text { chem }}}{\longrightarrow}$ & $8.6 \times 10^{-4}$ & $s^{-1}$ \\
\hline $\begin{array}{c}\text { Initiation by head-to-tail fission in } \\
\text { saturated chain }\end{array}$ & $P_{i+j} \stackrel{k_{I, H-T, \text { chem }}}{\longrightarrow} R_{i}+R_{j}$ & $2.0 \times 10^{-7}$ & $s^{-1}$ \\
\hline Depropagation by 6-scission & $R_{i} \stackrel{k_{d p, \text { chem }}}{\longrightarrow}$ & $5.0 \times 10^{3}$ & $s^{-1}$ \\
\hline Termination by recombination & $R_{i}+R_{j} \stackrel{<k_{t c, a p p}>}{\longrightarrow} P_{i+j}$ & $1 \times 10^{4}$ & $\mathrm{~mol} \mathrm{~L}^{-1} \mathrm{~s}^{-1}$ \\
\hline Termination by disproportionation & & $1 \times 10^{4}$ & $\mathrm{~mol} \mathrm{~L}^{-1} \mathrm{~s}^{-1}$ \\
\hline
\end{tabular}

an overall apparent activation energy is typically reported per peak but these values are far from fundamental. Pre-exponential factors are lacking almost entirely even on the formal level of a global kinetic model. Hence, one should realize that to increase our fundamental awareness for PMMA chemical recycling it is already a huge step forward if one focuses on the main elementary reactions and expected rate coefficients. In the present work, therefore only the core elementary reactions as proposed by Kashiwagi et al. ${ }^{16}$ are considered, as shown in Figure 3. Apart from three distinct degradation initiation reactions (yellow box), depropagation by 6 -scission (blue box) thus unzipping, and termination reactions are accounted for. For the latter, again both termination by recombination and disproportionation are considered (red box). 


\section{Degradation initiation reactions}

1) fission at unsaturated chain end

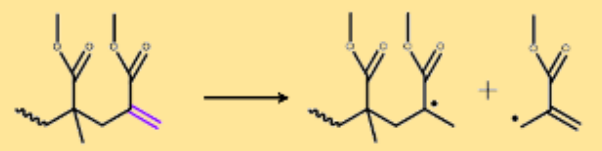

2) fission at head-to-head defect in a saturated chain

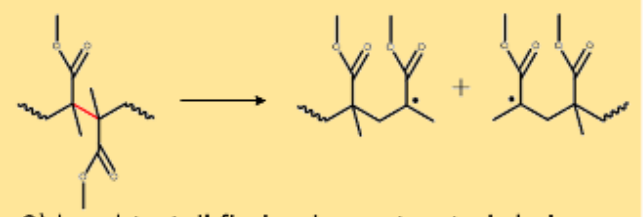

3) head-to-tail fission in a saturated chain

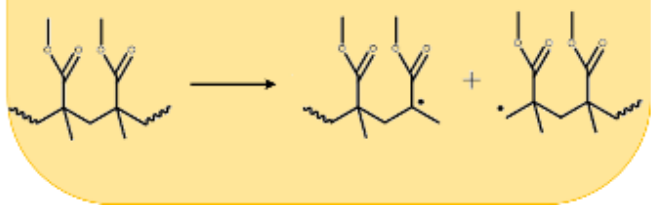

Depropagation reaction by betascission (unzipping)

$\beta$-scission

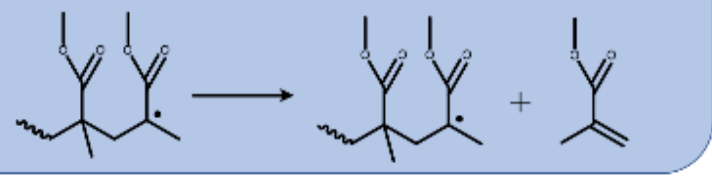

Termination reactions

1) by recombination
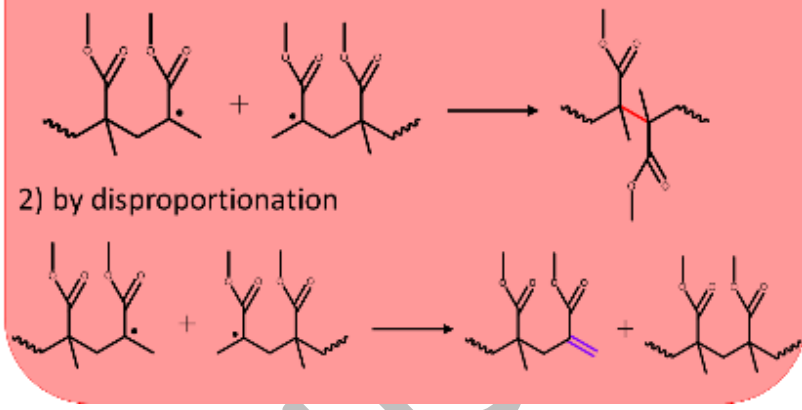

Figure 3: Basic reaction scheme to study thermal degradation of poly(methyl methacrylate) PMMA; kinetic parameters in Table 3 with the radicals only differentiated based on chain length; colors of defects/structural parameters same as in Figure 2.

The associated kinetic parameters for Figure 3 are given in Table 3, assuming formally a single phase reaction mixture at $623 \mathrm{~K}$ so that inherently no phase transfer phenomena need to be considered. For simplicity the primary and tertiary radicals, as formed via one of the three initiation reactions, are assumed to display an average reactivity and can be formally seen as an average species. This implies that the amount of possible termination products is reduced and also the switch from primary to tertiary radicals by 6 -scission of the side group resulting in the release of a methoxycarbonyl radical108 does need to be considered explicitly.

The relative order of magnitudes of the initiation reactivities in Table 3 are in line with literature data. ${ }^{11,12,16}$ For termination an average constant apparent reactivity $\left(\left\langle k_{t c / d, a p p}\right\rangle\right)$ is considered for simplicity thus no explicit calculation of this average is done in contrast to the FRP case above. This average value is significantly lower as the $k_{\mathrm{t} 11, \text { app }}$ value in Table 2 , as in a degradation experiment ones starts with a polymer-rich reaction mixture. For simplicity the contribution of both termination modes is taken equal, bearing also in mind the illustrative nature of the modeling of the second phase of the first test case. The key novelty of the present work lies in the generation of the correct starting MMD thus the one coming from the kinetic modeling of the prior FRP synthesis.

\section{Reactions and kinetic parameters for test case 2: phase 1: radical ring-opening copolymerization of MMA and MDO}

The bulk radical copolymerization of MMA and MDO is studied at $343 \mathrm{~K}$ selecting also AIBN as conventional radical initiator. As shown in Table 4, the basic reactions for a terminal radical copolymerization are considered differentiating between conventional radical dissociation, propagation and termination. For completeness the structural changes of the four propagation reactions are depicted in Figure 4(a). All rate coefficients are also mentioned in Table 4 and they are all literature based. ${ }^{26}$ Note that the associated monomer reactivity ratios $\left(r_{\mathrm{MMA}}=3.5\right.$ and $\left.r_{\mathrm{MDO}}=0.04\right)$ are very different, highlighting that both MMA and MDO ending radicals prefer propagation with MMA. Hence, under the selected batch conditions a depletion in MMA is expected. 
Table 4: Reactions for radical polymerization of methyl methacrylate (MMA) and methylene-1,3-dioxepane (MDO) at 343 K with 2,2'-dimethyl-2,2'-azodipropiononitril (AIBN) as conventional radical initiator. ${ }^{\text {a }}$ Also given are the kinetic parameters.

Equation $\quad k\left(\left(\mathrm{Lmol}^{-1}\right) \mathrm{s}^{-1}\right) \quad$ reference

\begin{tabular}{|c|c|c|}
\hline$I_{2} \stackrel{f_{\text {chem }}, k_{d, \text { chem }}}{\longrightarrow} 2 I$ & $0.75,2.5 \times 10^{-5}$ & 26 \\
\hline$I+M_{1} \stackrel{k_{p 11, \text { chem }}}{\longrightarrow} R_{1}$ & $1.5 \times 10^{3}$ & 26 \\
\hline$I+M_{2} \stackrel{k_{p 22, \text { chem }}}{\longrightarrow} R_{2}$ & $2.4 \times 10^{3}$ & 26 \\
\hline$R_{i, 1}+M_{1} \stackrel{k_{p 11, \text { chem }}}{\longrightarrow} R_{i+1,1}$ & $1.5 \times 10^{3}$ & \\
\hline$R_{i, 1}+M_{2} \stackrel{k_{p 12, \text { chem }}}{\longrightarrow} R_{i+1,2}$ & $\frac{k_{p 11, \text { chem }}}{r_{M M A}{ }^{a}}$ & 26,109 \\
\hline$R_{i, 2}+M_{1} \stackrel{k_{p 21, \text { chem }}}{\longrightarrow} R_{i+1,1}$ & $k_{p 22, \text { cher }}$ & 26,109 \\
\hline$R_{i, 2}+M_{2} \stackrel{k_{p 22, \text { chem }}}{\longrightarrow} R_{i+1,2}$ & $2.4 \times 10^{3}$ & 26,109 \\
\hline$R_{i, 1}+R_{j, 1} \stackrel{<k_{t d, a p p}>}{\longrightarrow} P_{i}+P_{j}$ & $1 \times 10^{7} \mathrm{~b}$ & 26,109 \\
\hline$R_{i, 1}+R_{j, 1} \stackrel{<k_{t c, a p p}>}{\longrightarrow} P_{i+j}$ & $1 \times 10^{7} \mathrm{~b}$ & 26,109 \\
\hline
\end{tabular}

a terminal reactivity ratios $r_{\mathrm{MMA}}=3.5$ and $r_{\mathrm{MDO}}=0.04^{109} ;{ }^{\mathrm{b}}$ literature value for recombination with radicals of length unity but for illustration purposes also taken for the other termination types/modes.

To simplify the kinetic description, taking into account the illustrative nature of this second test case, average but constant apparent reactivities are used as for the second phase of the first test case. A typical value of $10^{7} \mathrm{~L} \mathrm{~mol}^{-1} \mathrm{~s}^{-1}$ is considered consistent with previous studies in which such simplified approach to account for diffusional limitations on termination has also been considered. ${ }^{26,94}$ For the other reactions, diffusional limitations are ignored for simplicity.

\section{Reactions and kinetic parameters for test case 2: phase 2: degradation by hydrolysis of poly(MMA-MDO)}

For the kinetic description of the degradation of the MMA/MDO copolymer only the hydrolysis reaction is required. As shown in Figure 4(b) each MDO moiety can be hydrolyzed. In the presence of water, the ester group undergoes a transesterification, breaking the polymer chain into two fragments: one having a carboxyl group and the other a hydroxyl group. Hence, the hydrolysis aims at converting larger polymer chains into smaller functional oligomers which can ultimately be biodegraded by bacteria.

To a first approximation a constant MC hydrolysis rate coefficient is utilized. However, the actual value is irrelevant if one expresses the degradation process on an ester conversion basis, as performed in the present work. 


\section{a) Propagation reactions}

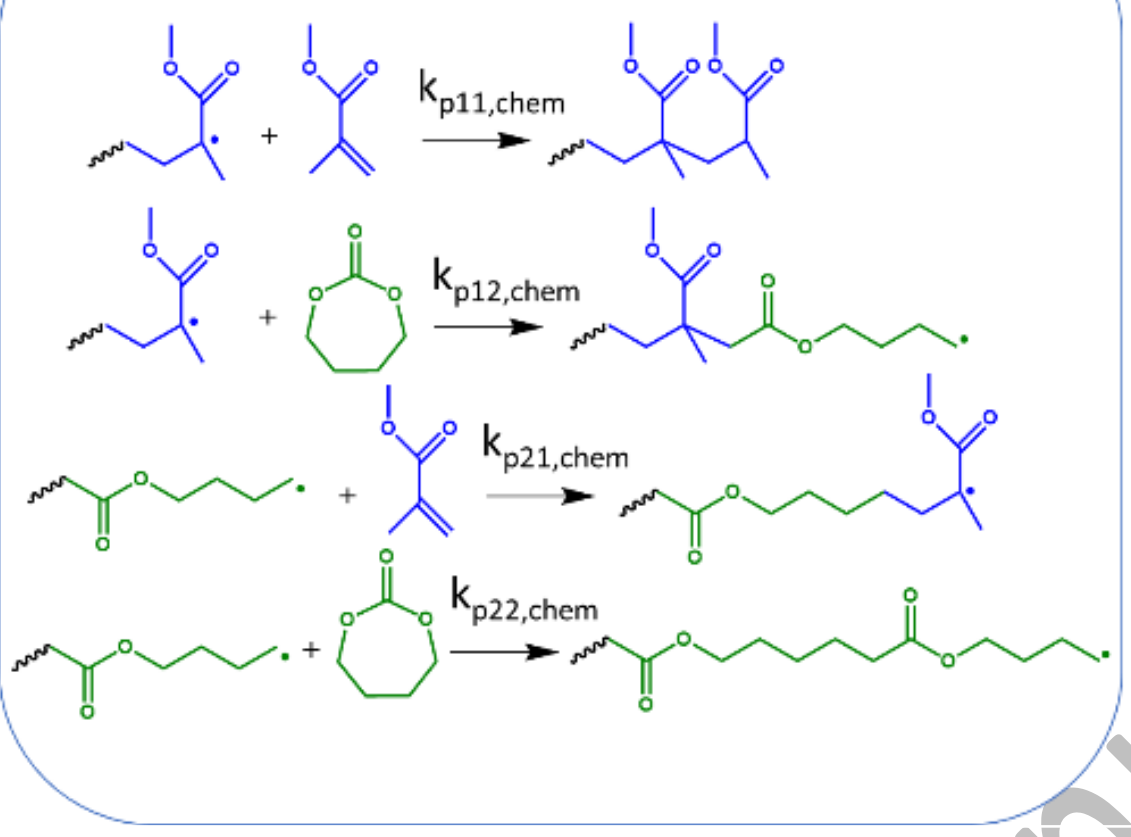

\section{b) hydrolysis}

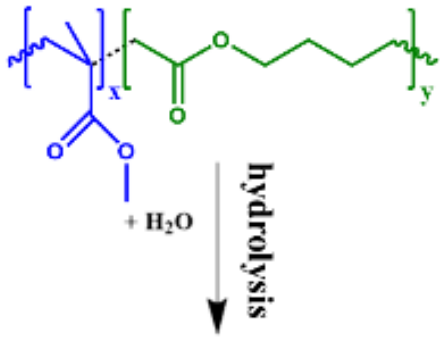<smiles>CCCCCCOC(=O)CC(C)(CC)C(=O)OC</smiles>

Figure 4: (a) Four propagation reactions for radical copolymerization of MMA and 2-methylene-1,3-dioxepane (MDO) assuming a terminal model to describe the propagation reactivity; (b) Hydrolysis reaction for a MDO moiety in a poly(MMA-MDO) chain.

\section{Results and discussion}

First the $k \mathrm{MC}$ modeling results are discussed for FRP of MMA including a benchmark with experimental literature data. Benefiting from the explicit visualization of the molecular build-up of individual chains as obtained at the end of the FRP synthesis it is then illustrated how the chemistry of thermochemical recycling of PMMA can be understood in detail. Specific focus is on the strength of the $k \mathrm{MC}$ platform to accurately represent the global shape of log-MMDs, covering strong tail variations and bimodal character. The general applicability of the $k \mathrm{MC}$ modeling tool is further highlighted focusing on radical copolymerization of MMA and MDO accompanied by a detailed computational analysis of the subsequent hydrolysis.

\section{Case study 1: FRP of MMA}

Bulk FRP has been studied intensively in the past decades. ${ }^{21,81,84,93-95,104,110}$ With MMA as monomer historically the main target has been on the simulation of average molecular properties as experimentally often recorded under isothermal conditions, as for instance done in the leading study by Hamielec and Balke. ${ }^{81,104,107,110}$ A first challenge remains the correct representation of diffusional limitations on termination. Most termination models are based on overall fitting parameters and have no direct link with the actual measurement of the chain length and monomer conversion dependencies of apparent termination rate coefficients. In this respect the more recent developments based on the RAFT-CLD-T technique ${ }^{82,97-99}$ are very relevant although they have been rarely combined with the actual description of FRP kinetics. ${ }^{98,111,112}$ The relevance of such combination was recently put forward by De Keer et al. ${ }^{111}$ for FRP of styrene, highlighting that only with such detailed RAFT-CLD-T parameters the bimodal character of the log-MMD and the impact of reaction conditions on the interplay of chain transfer to monomer/dimer and termination could be fully captured.

In the present work it is further confirmed that chain length and monomer conversion dependent RAFT-CLD-T parameters allow to accurately reflect the gel-effect, as for instance illustrated in Figure 6 focusing at a comparison of simulation and experimental results at $343 \mathrm{~K}$. The green full lines highlight an acceptable joint description of the experimental variation of the monomer conversion with time and the $x_{n}$ evolution with monomer conversion (green symbols), considering the literature based parameters in Table 1 and 2. Preference should here be first given to the correct description of the experimental monomer conversion data and then the experimental $x_{n}$ data, as the former are characterized by a much lower experimental error. This good match without additional tuning (condition from high temperature regime) is remarkable and highlights the overall strength of the use of the RAFT-CLD-T technique to describe the gel-effect. 
a)

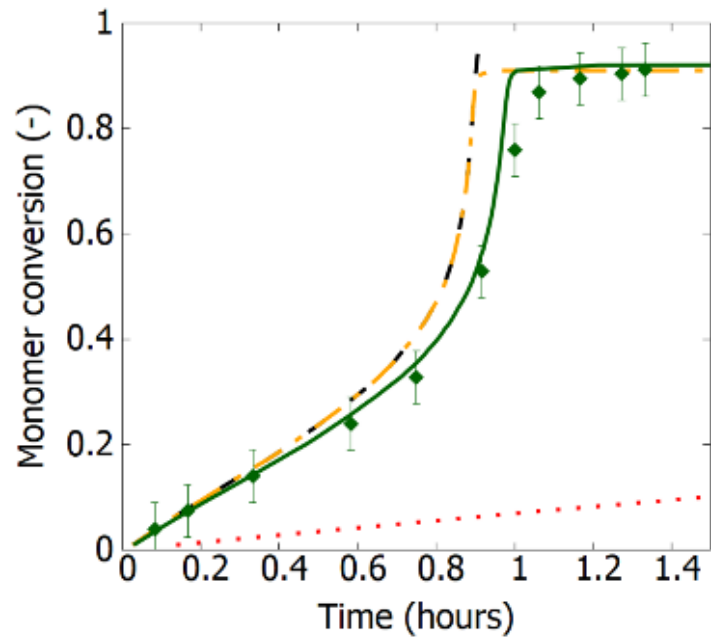

b)

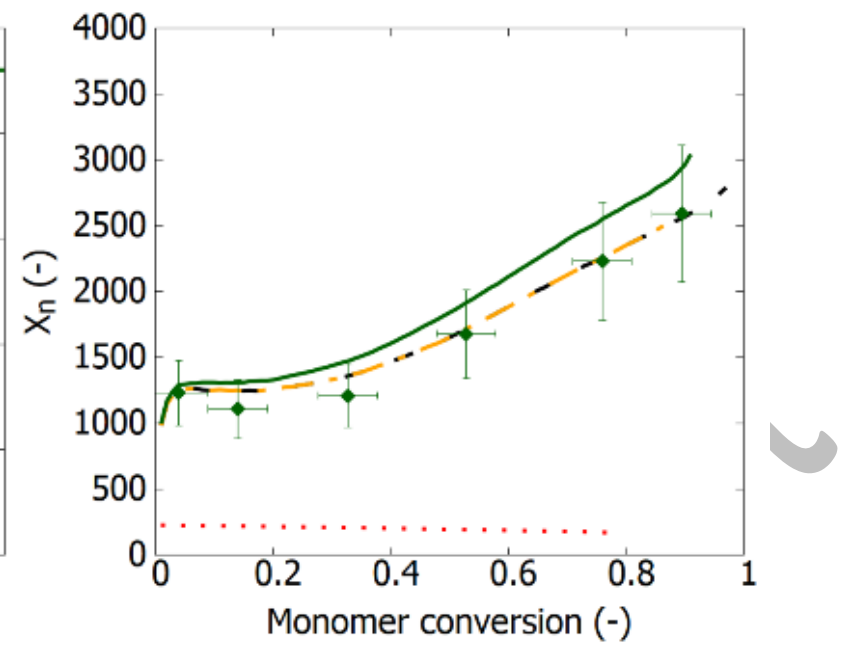

Figure 5: For bulk isothermal FRP of MMA ( $343 \mathrm{~K}$ and ([AIBN $]_{0}:[\mathrm{MMA}]_{0}=0.003$ ) the stepwise simulated inclusion of the cage, glass, and gel-effect for (a) the monomer conversion as a function of time and (b) the number average chain length $\left(x_{n}\right)$ as a function of monomer conversion; red dotted line: simulation without diffusional limitations (always $k_{\mathrm{t} 11, a p p}$ ); black dashed line: with incorporation of gel-effect; yellow dashed-dotted line: with incorporation of both gel and glass effect; green full line: incorporation of cage, glass, and gel-effect (parameters in Table 1 and 2). Green diamonds: experimental data points ${ }^{10}$

The simulated full green lines are not only affected by the gel-effect as not only diffusional limitations on termination matter but also those on propagation and conventional initiation, specifically from intermediate monomer conversions onwards, as illustrated by the other simulated lines in this figure and further confirmed in Figure S4 of the Supporting Information depicting the variations of the corresponding apparent (rate) coefficients. For completeness the dotted red line gives the simulations results at $343 \mathrm{~K}$ with only intrinsic kinetics thus no correction for diffusional limitations at all. This implies that for termination always the given $k_{\mathrm{t} 11, \mathrm{app}}$ value in Table 2 is utilized in the $k \mathrm{MC}$ model. From this simulation it becomes clear why previous studies have focused so heavily on the description of diffusional limitations, as the complete wrong simulated trend results. There is under such model assumptions no S-shaped curve for the monomer conversion and $x_{n}$ is always decreasing in contrast to what is observed experimentally in Figure 5(b). Furthermore, with only a gel-effect so with always a constant $k_{\mathrm{p}, \mathrm{chem}}$ as in Table 1 and considering a typical average low monomer conversion value of $0.58^{81}$ for $f_{\text {chem, }}$, the dashed black lines in Figure 5 result, which demonstrates a very strong impact of the gel-effect on both FRP characteristics. Less termination because of lower average apparent termination rate coefficients implies more propagation and thus an increase in both the simulated polymerization rate and $x_{\mathrm{n}}$ variation.

The additional consideration of the glass effect in Figure 5 (yellow dashed-dotted line) allows to explain the experimental limit on the highest achievable monomer conversion (value of $c a .0 .9$ ), as the propagation rate is strongly diminished toward the end of the polymerization because of a sudden drop of the apparent propagation rate coefficient. This combined consideration of the gel- and glass effect (yellow dashed-dotted line) although leads to a more pronounced increase of the simulated $X_{\mathrm{m}}$ at lower times in Figure 5(a) in contrast to what is experimentally observed. This mismatch for $X_{m}$ is overcome by also considering the cage effect (from yellow dashed-dotted to green full line), i.e. the decrease of $f_{\text {app }}$ from intermediate $X_{m}$ onwards. In practice, the reaction mixture becomes gradually more viscous for AIBN initiator fragments to escape their so-called solvent cage and instead they react more and more with each other to form inert side products. In the present work, the $f_{\text {app }}$ variation is evaluated based on the model equations put forward in the pioneering work of Buback et al. ${ }^{84}$ At low $X_{m}$ however this $f_{\text {app }}$ model gives a value of 0.50 (see Table 1 ), which is slightly lower than 0.58 which is the value used during the whole FRP for the previously discussed black dashed and yellow dashed-dotted simulated lines. With the implementation of $f_{\text {app }}$ the radical concentration thus decreases, explaining why $X_{m}$ increases more slowly and $x_{n}$ more strongly with an inclusion of the cage-effect (green full lines). At the highest monomer conversions, the glass-effect becomes very dominant and a cessation of the FRP takes place, despite that AIBN fragments can still be formed. 

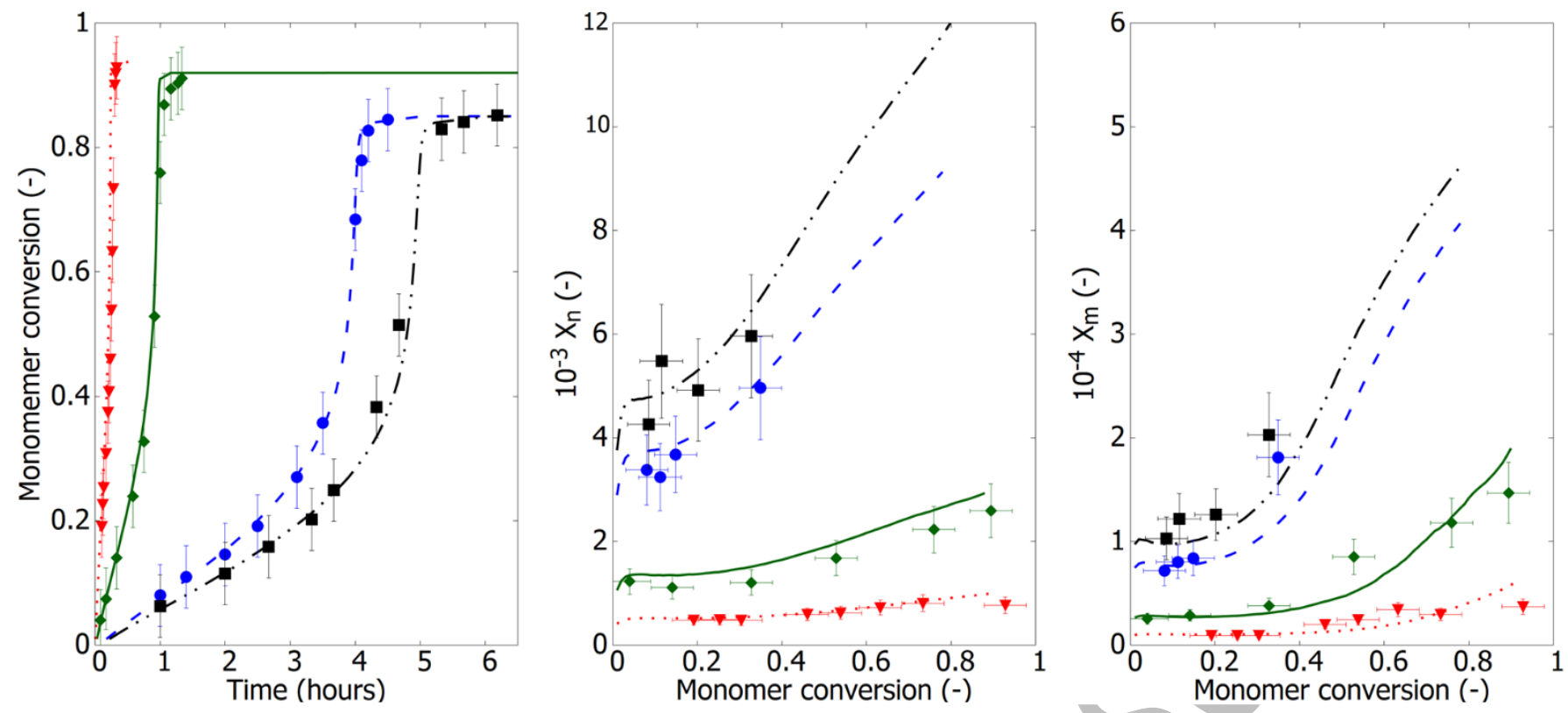

Figure 6: Benchmark of kinetic Monte Carlo ( $\mathrm{KMC}$ ) modeling results (lines) and experimental data ${ }^{92}$ (symbols) for bulk isothermal FRP of MMA under a broad range of initial conditions, considering (a) monomer conversion as a function of time, (b/c) number/mass average chain length ( $\mathrm{x}_{\mathrm{n} / \mathrm{m}}$ ) as a function of monomer conversion. Parameters in Table 1 and 2; red dotted line/triangles: $363 \mathrm{~K}$ and [AIBN] $]_{0}:[\mathrm{MMA}]_{0}=0.003$; green full line/diamonds: $343 \mathrm{~K}$ and $[\mathrm{AlBN}]_{0}:[\mathrm{MMA}]_{0}=0.003$ (as for Figure 6) blue dashed line/circles: $323 \mathrm{~K}$ and [AIBN] $]_{0}:[\mathrm{MMA}]_{0}=0.003$; black dashed-dotted-dotted line/squares: $323 \mathrm{~K}$ and $[\mathrm{AlBN}]_{0}:[\mathrm{MMA}]_{0}=0.002$. For guide-of-the eye typical average error bars are added.

The good benchmark between the $k \mathrm{MC}$ model and experiment is further confirmed in Figure 6 considering several temperatures $(323,343$ and $363 \mathrm{~K})$ and two initial AIBN concentrations ( 0.016 and $\left.0.027 \mathrm{~mol} \mathrm{~L}^{-1}\right)$. The prediction of the monomer conversion and $x_{\mathrm{n}}$ is good, whereas the prediction of $x_{\mathrm{m}}$ is somewhat less as is also the case in the modeling work of Achilias and Kiparissides ${ }^{81}$ considering the determinisic method of moments. This can be traced back to an original comment by Hamielec and Balke ${ }^{110}$ mentioning that the reproducibility of the central chromatogram heights was very good whereas the tails showed poor reproducibility. Hence, on an overall basis - thus considering the results in Figure 5 and 6 and the previous comment - it can be concluded that the $k \mathrm{MC}$ model is representative to describe FRP of MMA.

A closer inspection of Figure 6 reveals that by lowering the polymerization temperature and/or decreasing the initial AIBN amount much higher average chain lengths are obtained at the expense of polymerization time and limiting monomer conversion. Under such conditions diffusional limitations are also stronger as shown in Figure S5 of the Supporting Information. A stronger drop of the averaged apparent rate coefficients for termination and propagation is observed, explaining in Figure 6 the stronger increase at lower monomer conversions for $x_{n}$ and $x_{m}$ (stronger gel-effect) and the lower limiting conversion (stronger glass effect). A lower temperature also implies lower initial $f_{\text {app }}$ values and stronger decreases, further supporting these trends of a lower polymerization rate and the formation of longer chains at the larger monomer conversions. This again highlights the need of kinetic models fully accounting for the interplay of chemistry and diffusional limitations.

The strength of a $k \mathrm{MC}$ model is that it allows to obtain all molecular information at any time in the synthesis at any reaction condition thus also the full details regarding chain length characteristics at the limiting conversions at the lower polymerization temperatures and initial AIBN amounts. As shown in Figure 6(b) and Figure 6(c) at such limiting monomer conversions (black dashed dotted and blue dashed lines) no experimental $x_{n}$ and $x_{m}$ values could be recorded but the $k M C$ model could still be run. Moreover, this kinetic model gives access the the complete (log-)MMD and also information on the concentration and location of functional groups and structural defects. It should be stressed that older kinetic models revolving around the method of moments are typically only devoted to the calculation of averages and thus not such detailed molecular distributions.

For example, as shown in Figure 7(a), focusing on the bulk isothermal FRP of MMA at a low polymerization temperature of $323 \mathrm{~K}$ with $[\mathrm{AIBN}]_{0}=0.027 \mathrm{~mol} \mathrm{~L}^{-1}$, the $\mathrm{kMC}$ model is capable to depict at selected monomer conversions the log-MMD. Emphasis is here on a $X_{m}$ of 0.1 (blue line), 0.5 (black line) and 0.85 (red line), the latter being the simulated limiting conversion. It follows that there is a strong shift towards higher molar mass with monomer conversion, as is also confirmed by the strong increase of the $x_{n}$ value in Figure 6(b). This tendency of long chain formation even results in a higher molar mass peak at high monomer conversion in Figure 7(a), which again stresses the importance of the gel-effect and diffusional limitations in general. Due to the strong drop in the apparent termination rate, the macroradicals are at one point able to grow significantly longer and this results in a separate polymer population with higher molar mass. 
a)
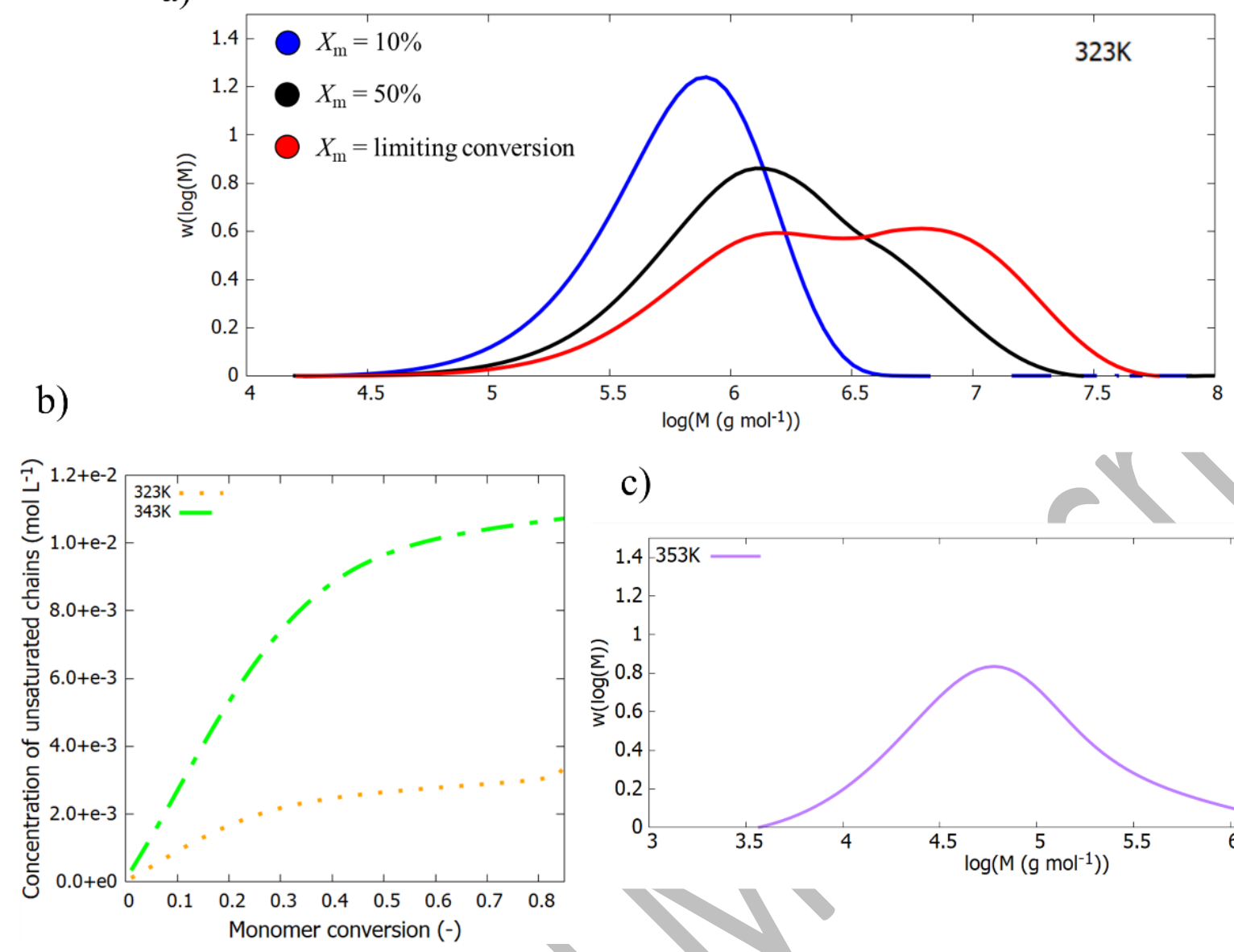

c)

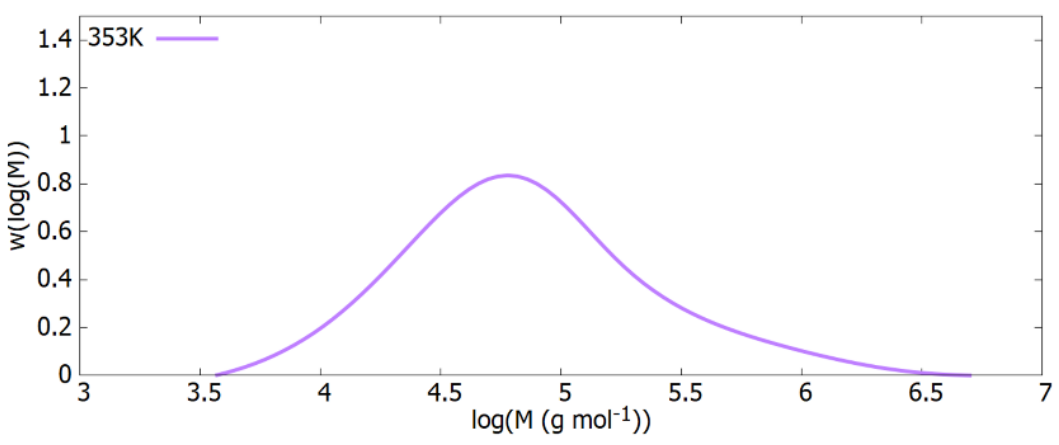

Figure 7: Kinetic Monte Carlo (KMC) model derived log - molar mass distribution (MMD) and information on functionalities/structural defects at any time during FRP of $M M A$ at any initial condition. (a) log-MMD at a monomer conversion of 0.1 , 0.5 and 0.85 (the limiting one) for $323 \mathrm{~K}$ and $[\mathrm{AlBN}]_{0}=0.027 \mathrm{~mol} \mathrm{~L}^{-1}$; (b) unsaturations as formed by termination by disproportionation and involving chain transfer to monomer as a function of monomer conversion for 323 and $343 \mathrm{~K}$ with $[\mathrm{AlBN}]_{0}=0.027$ mol parameters from Table 1 and Table 2 .

Furthermore, Figure 7 (b) illustrates the evolution of the concentration of chains with unsaturations either formed by termination by disproportionation or involving chain transfer to monomer, considering FRP of MMA at 323 (orange dotted line) and $343 \mathrm{~K}$ (green dashed dotted line) with $[A I B N]_{0}=0.027 \mathrm{~mol} \mathrm{~L}^{-1}$. It can be seen that upon progression of the polymerization the formation of this double bound functionality increases. More formation of this functionality implies more shorter chains and thus the number of unsaturations is inversely proportional to $x_{n}$ (see Figure 6(b)). Also here the effect of diffusional limitations comes into play. On the one hand termination by disproportionation is suppressed, leading to less functionality formation, but on the other hand chain transfer to monomer is more likely as chains are longer active. At the higher monomer conversions the monomer becomes although depleted, explaining the less pronounced increases at larger reaction times for both lines in Figure 7(b), with in any case larger values at the higher temperature (green line) as then the relative importance of chain transfer to monomer and termination by disproportionation is higher.

Figure 7(c) (purple full line) in turn shows the log-MMD at limiting conversion for a general reaction condition not included in Figure 6: $353 \mathrm{~K}$ with $[\mathrm{AIBN}]_{0}=0.17 \mathrm{~mol} \mathrm{~L}^{-1}$. It follows that again a distribution of bimodal nature is obtained with an elongated tail, although less pronounced due to a weaker gel effect at higher temperatures. Compared to Figure 7(a) this log-MMD is relatively shifted more to the lower molar mass region as well. Hence, depending on the reaction conditions the shape and positioning of the log-MMD is completely different, highlighting the need of a detailed kinetic modeling approach, as demonstrated in the present work. In any case, the tail of the distrubution is well-presented.

Figure 8 (bottom; left subplot) highlights the strenght of the explicit vizualization of individual PMMA chains with $k M C$ modeling at limiting conversion (0.85) for the condition with $343 \mathrm{~K}$ and $[\mathrm{AIBN}]_{0}=0.027 \mathrm{~mol} \mathrm{~L}^{-1}$ (covered in Figure 5 and 6 ). The black color highlights the presence of a MMA segment and the other colors a specific structural defect or functionality. Focus is only on ca. 200 chains (although the $k \mathrm{MC}$ model considers much more chains: up to $10^{8}$ ) and the first part of the molecular structure of these chains (focus only up to a chain position of 100 although the maximum chain length can be as high as ca. 15000). It follows that most chains have been initated by an AIBN fragment (blue color at first position) and rarely another 
In any case, the tail of the distrubution is well-presented.
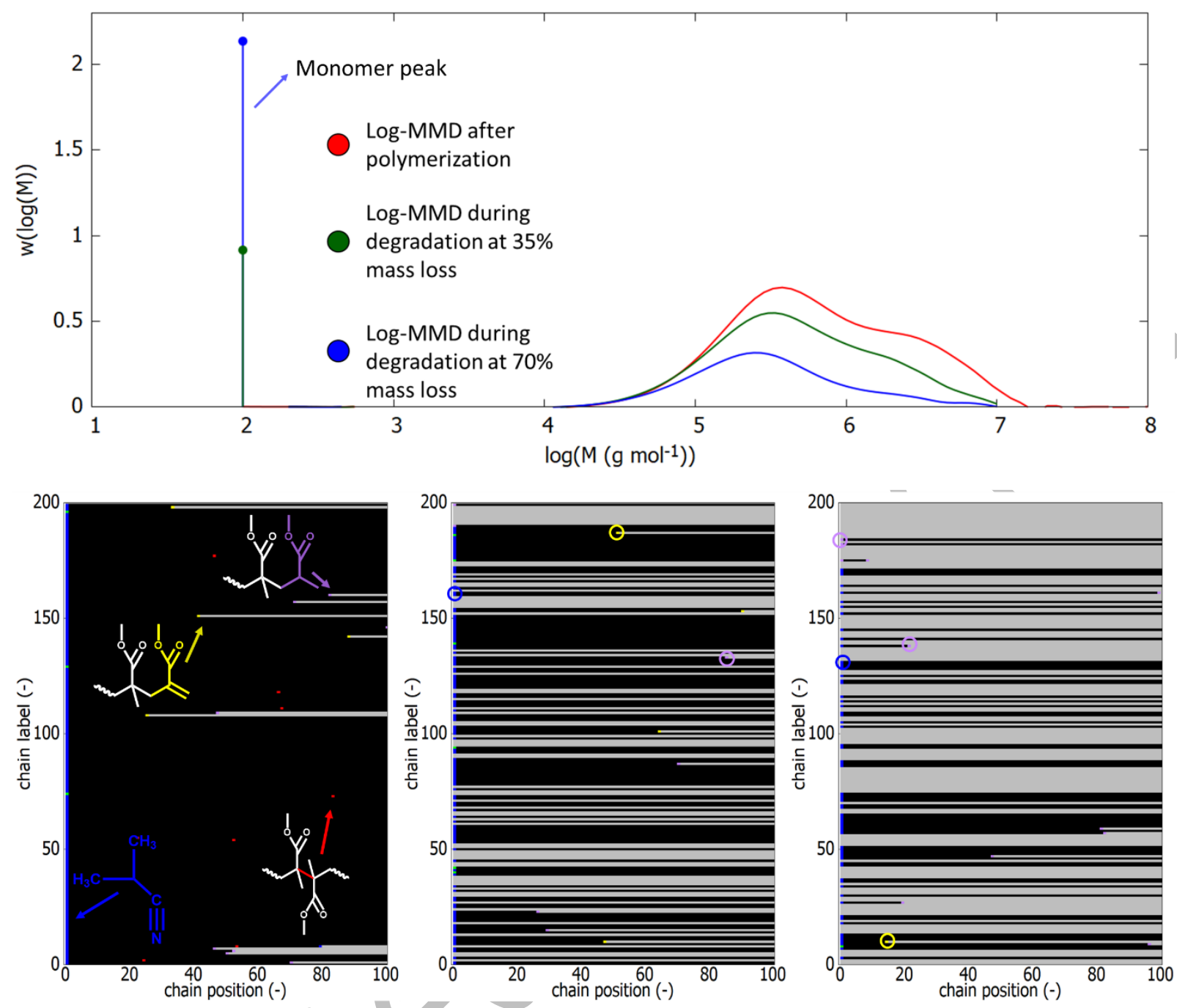

Figure 8: Top: simulated log-molar mass distributions (MMDs) (with spline correction) for lab-scale thermochemical recycling of FRP-made PMMA at 625 K at a mass loss percentage of $0 \%$ (synthesis up to limiting conversion as in Figure $5 ; 343 \mathrm{~K}$ ), $35 \%$ and $70 \%$; also included MMA formation (log(M) $=2$ ). Bottom: explicit visualization focusing on a limited number of chains and only the first 100 chain positions (left to right 0,35 and $70 \%$ mass loss), select ing grey as background; in the left subplot the by disproportionation or chain transfer to monomer, yellow: unsaturated MMA unit by termination by disproportionation and green: unsaturated chain end resulting from chain transfer to monomer.

chain initiation has taken place (e.g. almost no green color at the first position, which is indicative of transfer to monomer). At random positions one can further notice head-to-head defects (red color) and occasionally an unsaturation (yellow color). Hence, the current explicit molecular information is useful to fundamentally capture a thermal degradation, as covered in the next subsection.

\section{Case study 1: Subsequent thermochemical recycling toward MMA recovery}

The degradation process of FRP-made PMMA is studied at $625 \mathrm{~K}$ with the kinetic parameters mentioned in Table 3 and focusing first on the bulk FRP reference condition introduced before (Figure 5): $343 \mathrm{~K}$ and ([AIBN $]_{0}:[\mathrm{MMA}]_{0}=0.003$ ). The PMMA reactant is considered to be the polymer as synthesized at the limiting monomer conversion. Figure (top) shows as a red full line the corresponding in silico generated bimodal FRP log-MMD. Also included in this subfigure are the simulated log-MMDs once the mass loss due to thermal degradation is equal to $35 \%$ (green line; middle distribution) and $70 \%$ (blue line; left distribution). The $k M C$ model clearly predicts that initially the main peak of the log-MMD decreases and then slighly shifts to the left, although the chains with the lowest molar masses remain almost unattacked. The polymer mass loss is formally captured by introducing a monomer signal at $\log (M)=2$, which is close to the molar mass of MMA. The computation times are ofcourse very dependent of the reaction volume, for these simulations with a reaction volume of $2.0 \times 10^{-15} \mathrm{~L}$ the simulation times are about 8 hours $(4$ hours for the polymerization and 4 hours for the degradation). 
The subplots at the bottom of Figure 8 show the explicit visualization of 200 chains as selected out of the main sequence matrix and depicted as black lines on a grey background accounting for the values in the segment length matrix. The $x$-axis indicates the position in the polymer backbone as restricted to 100 just for illustration purposes although its actual maximum number can be as high as $c a$. 15000 representing the maximum chain length. The $y$-axis in Figure 8 (top plot) highlights in turn the row/label number at which the chain is present in the sequence matrix. As these chains are each time randomly selected and breaking by fission so that their fragments need to be re-stored at new rows, the label $y^{*}$ (e.g. 50) in these three subplots is likely not referring to the same chain (fragment). Note that once a chain (fragment) is fully degraded it becomes a grey line but formally can still be sampled, explaining why the number of black lines is decreasing from left to right at the bottom of Figure 8 . Also included in these subplots are the colors introduced in Figure 2 to highlight the presence of certain functional groups or structural defects, due the occurrence of certain reactions. For ease of readibility in the left subplot explicit chemical structures are depicted in the same colors as well.

As already highlighed in the discussion of the PMMA synthesis, the left subplot at the bottom of Figure 8 ( $0 \%$ mass loss) is characterized by head-to-head defects (red dots). These defects are the most prone to initiate a degradation mechanism consistent with the highest initiation rate coefficient in Table 3 (first row), which is so high that it compensates for these defects being present in undersize compared to monomer units with many head-to-tail bonds thus many locations for head-to-tail fission. It follows from the middle subplot at the bottom of Figure 8 (35 m\% loss) that indeed such defects quickly disappear, as they are not present anymore. Moreover, in the right subplot at the bottom of Figure 8 (mass loss of $70 \%$ ) they cannot be identified. Since these headto-head defects are exclusively formed through termination by recombination of two macroradicals in the prior synthesis, these chains occupy the larger molar mass region so that it can be understood why the high molar mass peak in the log-MMD (Figure 8; top) strongly decreases so that the distribution is more located at lower molar masses in the first $35 \%$ of mass loss.

At higher mass losses (35-70\% mass loss) there is thus a larger dominance of the other two initiation mechanisms in Figure 3 although the parameters in Table 3 indicate that there is preference for fission at an unsaturated chain end. The presence of such unsaturations is confirmed in the middle and right subplot by for instance the yellow squares representing terminal vinyl bonds as originating from termination by disproportionation during the preceding FRP or as formed during the thermal degradation. However, the number of monomer units is high with respect to the number of unsaturations so that both degradation phenomena are taking place from a kinetic point of view. Upon degradation species can ultimately become MMA, explaining the increase of the signal at $\log (M)=2$ in the top of Figure 9 . In addition, also more colors can be identified in the bottom plots of Figure 8 for the first positions. At the start one has dominantly blue (AIBN fragment) and occasionally green (chain transfer to monomer fragment) but later on one also has for instance purple. Due to chain fission the "right" saturated ends due to termination by disproportionation or chain transfer to monomer become "left" chain ends as the radical centre is always plotted at the "right" end of the chain. To give the reader some idea of how the quantity of structural defects and functional groups evolves during both polymerization and degradation we refer to Figure S6 in the Supporting Information. The Figure S6 is similar to Figure 7(b) (where the evolution of the unsaturated chain concentration is shown during FRP of MMA), however here the evolution of all relevant functional groups/defects are shown both during polymerization and subsequent degradation.

The thermal degradation at $625 \mathrm{~K}$ is also modeled for a significantly different starting PMMA as made through FRP at higher temperature and initial AIBN concentration $\left(363 \mathrm{~K}\right.$ and $\left.[\mathrm{AIBN}]_{0}=0.17 \mathrm{~mol} \mathrm{\textrm {L } ^ { - 1 }}\right)$. Such conditions result in a polymer with a much lower average chain length and thus a log-MMD that is more shifted towards the left (see red line in top of Figure 9), due to the higher radical concentration and the higher (apparent) termination rate during the synthesis. Also the dispersity of this initial distribution in Figure 9 (top) is much lower $(\theta=2.39)$ compared to the dispersity of the polymer in Figure $8(\theta=6.29)$. With elevated temperature and initial AIBN concentration the gel-effect is less present and the bimodal character for the log-MMD is rather minimal. This limited impact of larger chains also follows from the left bottom subplot in Figure 9 with more grey background, implying that very short chains are present already at the start with e.g. chain ends formed by termination by disproportionation (yellow dots).

As the number of monomer units is on average lower per chain and head-to-head defects are present in many chains again the degradation mechanism starts with a dominant role for initiaton by head-to-head fission. Here the tail of the log-MMD is also degradated strongly at the start as on a relative basis still many long chains are existing with such head-to-head defects. The corresponding log-MMD at 35\% mass loss is depicted as green dots in Figure 9 (top) and upon comparison with the log-MMD at $70 \%$ mass loss (blue dots in the same subfigure) a quite regulated degradation pattern can be identified, which is an indication that the chain length of unzipping can be similar to the average chain length of the polymer chains. If this is not the case termination occurs so that smaller dead PMMA chains are formed, shifting the log-MMD to the left. On a log-scale this effect seems small but on normal scale it still relates to a lowering with 1000 monomer units. This thus highlights the relevance of parameter tuning of elementary rate coefficients for thermal degradation in future studies. 


\section{Case study 2: radical copolymerization of MMA and MDO}

The developed matrix-based $k M C$ platform can also be applied for radical copolymerization. In the present work this is illustrated for batch radical copolymerization of MMA and MDO at $343 \mathrm{~K}$, selecting again AIBN as conventional radical initiator. The kinetic parameters for the simulations are taken from Table 4 and are all literature based. Figure S7 of the Supporting
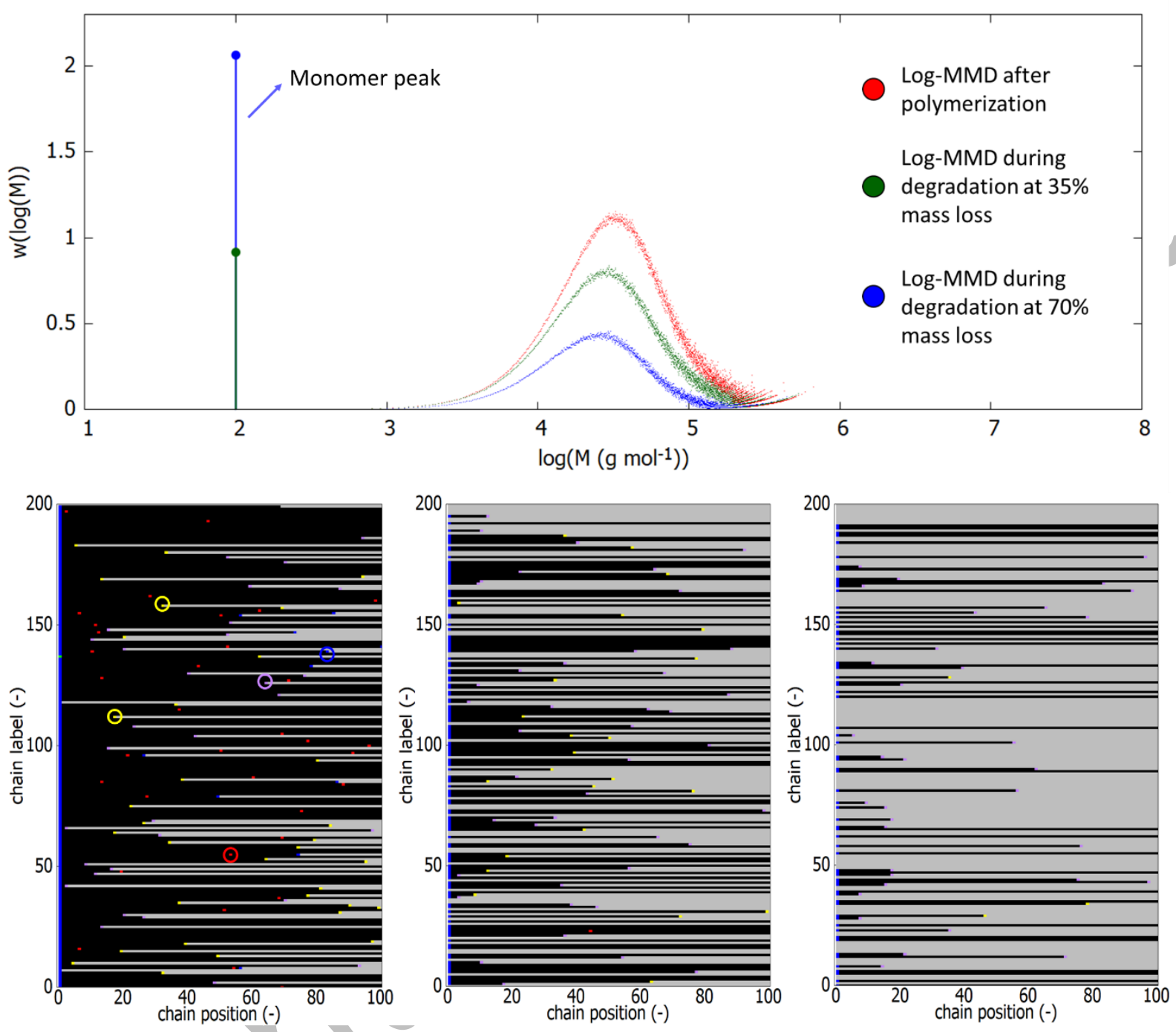

Figure 9: Top: simulated log-molar mass distributions (MMDs) (without spline correction) for lab-scale thermochemical recycling of FRP-made PMMA at 625 K at a mass loss percentage of $0 \%$ (synthesis up to limiting conversion for $363 \mathrm{~K}$ and $\left.[\mathrm{AIBN}]_{0}=0.17 \mathrm{~mol} \mathrm{~L}^{-1}\right), 35 \%$ and $70 \%$; also included $\mathrm{MMA}$ formation at ca. log( $\mathrm{M}$ ) $=2$. Bottom corresponding explicit visualizations focusing on a limited number of chains and only the first 100 chain positions.

Information highlights the variation of the overall monomer conversion (thus for both comonomers together) as a function of time for an equimolar monomer feed composition $\left(f_{\mathrm{MMA}, 0}=f_{\mathrm{MDO}, 0}=0.5\right)$. In the same figure, the variation is given of $x_{\mathrm{n}}$ and $\Theta$ as a function of this overall monomer conversion. It follows that the polymerization is finalized after ca. 4.5 hours with a final $x_{\mathrm{n}}$ and $\bigoplus$ value of ca. 107 and 4.2. For simplicitiy, as explained above, no cage and glass effect are here considered so that the FRP is only slowed down upon monomer depletion.

Figure 10(a) (middle lines) depicts the corresponding variation in instantaneous (average or overall) copolymer composition

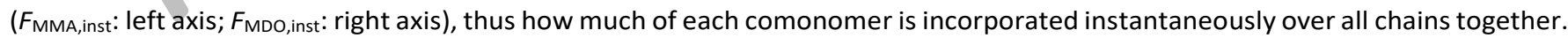
Besides considering the situation of $f_{\mathrm{MMA}, 0}=f_{\mathrm{MDO}, 0}=0.5$ also the situations of $f_{\mathrm{MMA}, 0}=0.75 ; f_{\mathrm{MDO}, 0}=0.25$ and $f_{\mathrm{MMA}, 0}=0.25 ; f_{\mathrm{MDO}, 0}=0.75$ are included. Note that the initial $F_{\text {inst }}$ values in this subfigure can also be assessed by applying the terminal Mayo-Lewis equation: ${ }^{113}$

$$
F_{M M A, \text { inst }}=\frac{r_{M M A} f_{M M A}^{2}+f_{M M A} f_{M D O}}{r_{M M A} f_{M M A}^{2}+2 f_{M M A} f_{M D O}+r_{M D O} f_{M D O}^{2}}=1-F_{M D O, \text { inst }}
$$

This formula gives for $F_{M M A, i n s t}$ values of $0.919,0.812$ and 0.659 (black spheres in Figure 10(a)) for the three situations according to decreasing initial MMA amount. The $k M C$ simulations give values of $0.918,0.811$ and 0.656 at $1 \%$ overall monomer conversion, highlighting the consistency of the developed framework. These data also allow to deduce that $f_{\mathrm{MDO}, 0}$ has to be sufficiently large 
to garantuee the satisfactory incorporation of $\mathrm{MDO}$ in the chains. For example, with $f_{\mathrm{MDO}, 0}=0.75$ the (co)polymer chains formed at the start only consist for ca. 35\% out of MDO units. It further follows that in each of the 3 situations one observes so-called composition drifting ${ }^{114-117}$ with the monomer mixture being depleted in MMA so that chains formed later on are more rich in MDO units. For example, for $f_{\mathrm{MMA}, 0}=f_{\mathrm{MDO}, 0}=0.5$, the chains contain as much as $80 \% \mathrm{MMA}$ units at low overall monomer conversions, while polymer chains formed at high overall monomer conversion $(>0.80)$ contain no MMA units at all. For the situation with less MMA at the start $\left(f_{\mathrm{MMA}, 0}=0.25 ; f_{\mathrm{MDO}, 0}=0.75\right)$ this switch to a dominance of MDO incorporation understandably takes place at a lower overall monomer conversion of 0.60 and for the

a)

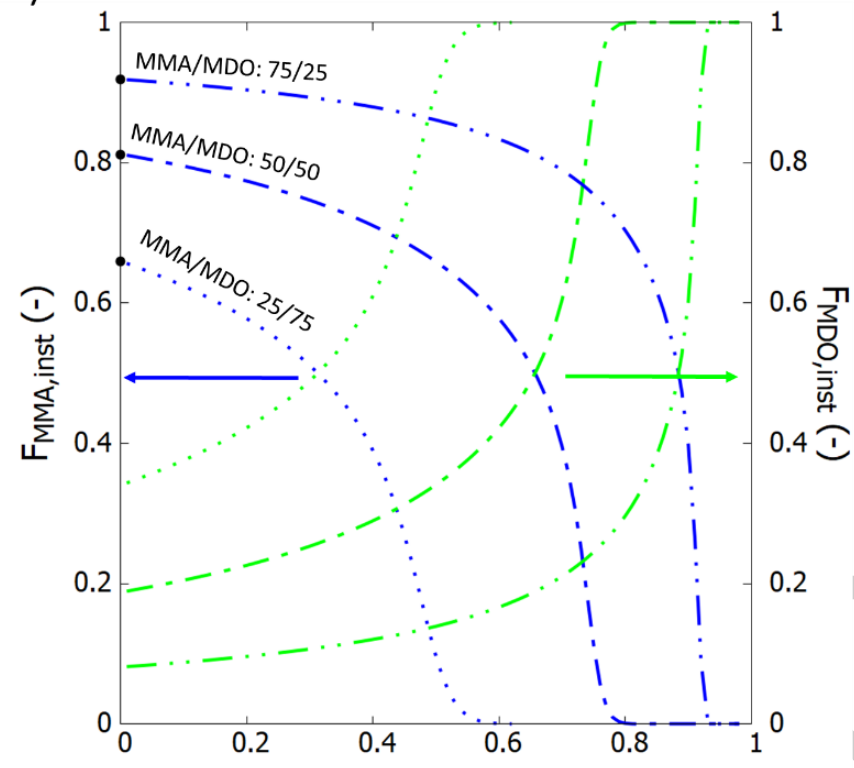

Overall monomer conversion (-) b)
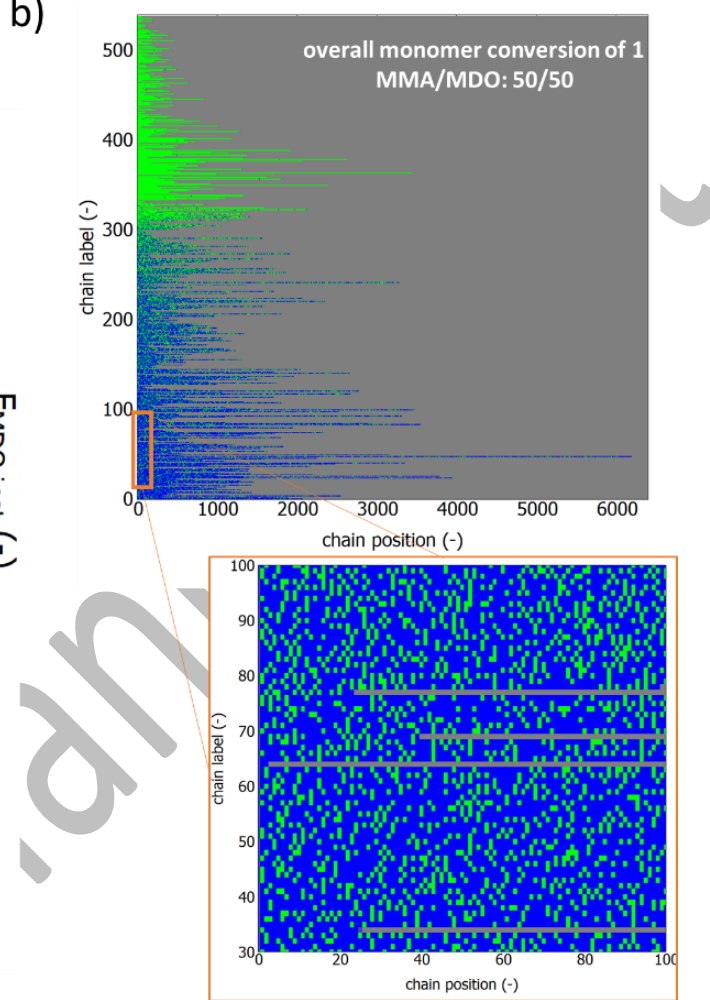

Figure 10: (a) Simulated instantaneous (average/overall) copolymer compositions ( $F$ values) as a function of overall monomer conversion for batch radical

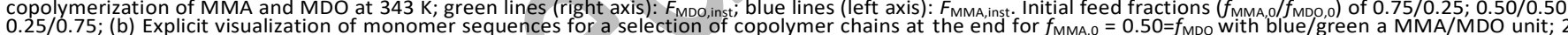
$0.25 / 0.75$; (b) Explicit visualization of monomer sequences for a selection of copolymer chains at the end for $f_{\text {MMA, } 0}=0.50=f_{\text {MDO }}$ with blue/green a MMA/MDO unit; 2
mol\% AlBN initially; model parameters: Table 4; black spheres in (a) highlight terminal Mayo Lewis values (Equation (1)); in (b) the chains depicted at the bottom are formed a lower overall monomer conversion and those at the top at higher overall monomer conversion due to a regulated sampling of a representative number of chains out of a total chain population of $10^{7}$ chains.

situation with more $\mathrm{MMA}$ at the start $\left(f_{\mathrm{MMA}, 0}=0.75 ; f_{\mathrm{MDO}, 0}=0.25\right)$ this switch is still unavoidable, with the switch occurring at an overall monomer conversion of $c a$. 0.80 . Hence, for any situation, the instantaneous comonomer incorporation rates are strongly time depending so that a very strong intermolecular compositional heterogeneity results. ${ }^{118}$

This inherent heterogeneity for (batch) radical copolymerization affects the efficiency of subsequent degradation/recycling processes. Specifically for the MMA/MDO system under investigation this leads to an important design criterion in view of ease of degradability. The MMA segments that are formed the earliest will be the longest, possibly too long to be biodegraded by biota later on. This is aptly illustrated in Figure 10(b), which shows the explicit visualization of individual monomer sequences of a representative number of copolymer chains as present at final overall monomer conversion $\left(f_{\mathrm{MMA}, 0}=f_{\mathrm{MDO}, 0}=0.5\right)$, with green being a MDO unit and blue a MMA unit. Note that the chain labels on the $y$-axis are sampled chronologically during the $k M C$ simulation of the radical copolymerization, hence, the higher they are located in the subplot the more likely they present chain formation at a higher overall monomer conversion. This boils down to the fact that the chains at the bottom represent chains formed at low overal monomer conversion whilst chains at the top are formed close to an overall monomer conversion of 1.

It is clear from Figure 10(b) that the compositional heterogeneity of the copolymer product is high if the whole overall monomer conversion range is considered. Consistent with the trends in Figure 10(a) it is observed that the longest MMA segments are indeed formed at the beginning, as witnessed by the long blue segments at the lower part of the visualization plot. These are exactly the oversized segments which form the bottleneck for a successful degradation, as will covered later on. At high overall monomer conversions the chains are much shorter due to monomer depletion and they are becoming in the limit more "green" MDO homopolymer chains thus off-spec with respect to the target of the synthesis of PMMA-like chains. This explains why it has been recommended to not conduct the polymerization up to the end but to stop earlier to recover the unreacted MDO monomer. ${ }^{26}$ 
Post-processing of explicit vizualizations allows to simulate SLDs (see examples in Figure S8 of the Supporting Information) and average segment lengths as well. To ensure that the predicted segment lengths are reliable, we have benchmarked the model predictions at 1\% overall monomer conversion (values of 11.3/1.01, 4.44/1.04 and 2.14/1.10; MMA/MDO; again for the three situations according to decreasing initial MMA amount) against the ones accessible by applying the Mayo and Lewis terminal model (values of 11.5/1.01, 4.50/1.04 and 2.17/1.12; Equation S12 of the Supporting Information). Constitent data are again obtained as also further deducible by focusing at the

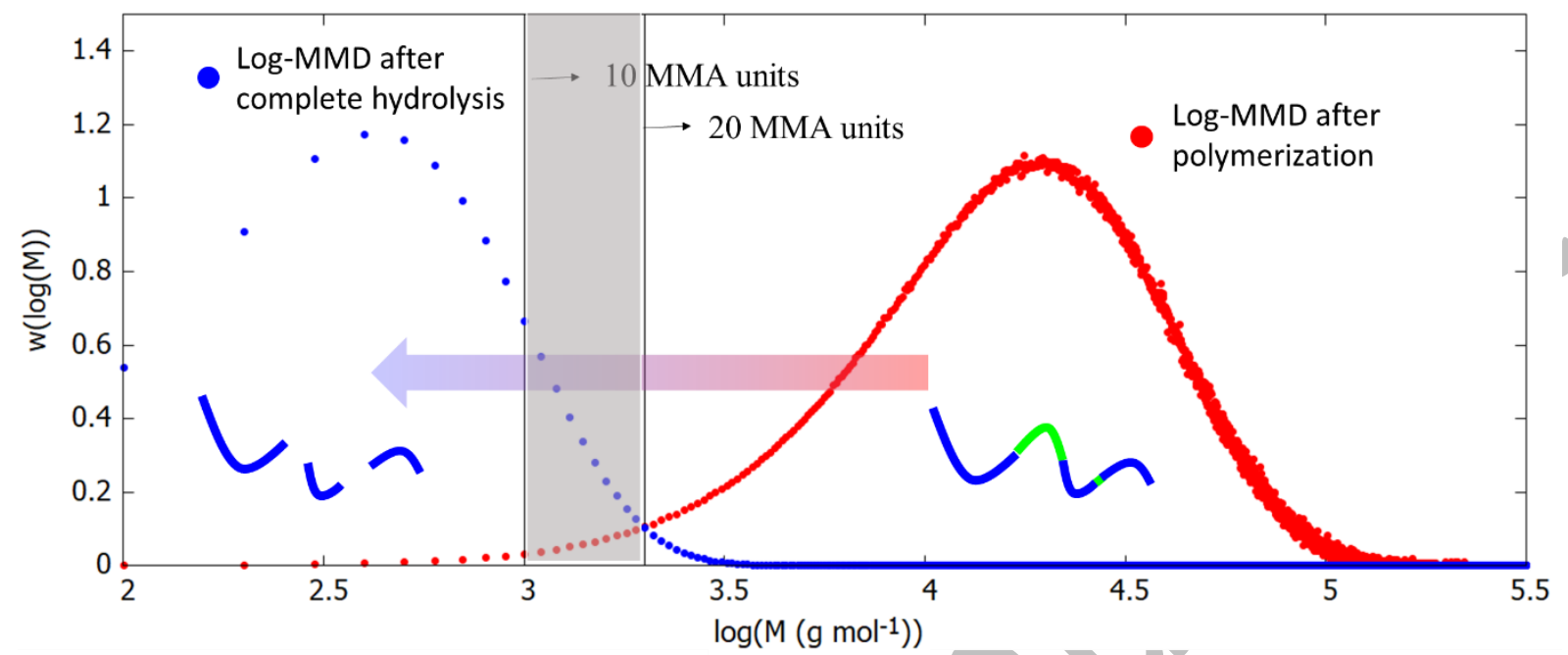

Figure 11: Red line: simulated log-molar mass distribution (log-MMD) for poly(MMA-MDO) corresponding to Figure 12 (left); Blue line: corresponding long-MMD after complete hydrolysis; the black demarcation lines indicate the molar masses of chains with 10 and 20 MMA units.

zoom at the bottom of Figure 11 (b) with for $f_{\mathrm{MMA}, 0}=0.5 ; f_{\mathrm{MDO}, 0}=0.50$ the initial $\mathrm{MMA} / \mathrm{MDO}$ segment lenghts indeed on average equal to $4 / 1$.

Hence, the $k M C$ model is capable to correctly predict the copolymer composition and segment lengths for the copolymerization of MMA and MDO, which are the most important molecular characteristics to predict the degradation by hydrolysis covered in the next subsection.

\section{Case study 2: subsequent hydrolysis of MMA-MDO copolymer}

One way to improve the biodegradability of vinyl polymers is the insertion of chemically less stable ester linkages in the polymer backbone during the synthesis, ideally each $10^{\text {th }}$ or $20^{\text {th }}$ monomer unit. ${ }^{27,28}$ Upon hydrolyis (Figure 5 (b)) such (co)polymers chains are converted into functional oligomers which can either be upcycled to new polymer products or biodegraded by bacteria. However, as vizualized in Figure 10 (b) $\left(f_{\mathrm{MMA}, 0}=f_{\mathrm{MDO}, 0}=0.5\right)$, these ester linkages are not included exactly every $10^{\text {th }}$ or $20^{\text {th }}$ unit in the backbone. Instead, severe compositional drifting occurs on top of the inherent random character of the propagation steps of Figure 4(a), as explained above.

The explicit visualization of the hydrolysis process considering as starting point the copolymer as made upon using $f_{\mathrm{MMA}, 0}=f_{\mathrm{MDO}, 0}=0.5$ and a final overall monomer conversion of 1 is shown in Figure S9. Hence, the left subplot (red box) in Figure S9 is the same as the vizualization plot shown in Figure 10(b). The right plot in Figure S9 shows the completely degraded product with the blue segments representing the remaining MMA-based oligomers. The figure in the middle gives the vizualization at which $50 \%$ of the ester linkages have been hydrolysed, as indicated by the white spaces on the grey original background. The subplots at the bottom a re a zoom-in of the chains with a chain label between 200 and 300 to showcase the evolution of these chains more clearly during the hydrolysis and to have a better view of the length of both the MMA and MDO segments.

Figure 11 shows the corresponding log-MMDs before (red line) and after complete (blue line) hydrolysis, including a first vertical black demarcation line around $\log (M)=3.3$, corresponding to a chain with $20 \mathrm{MMA}$ units and a second such $\operatorname{line}$ at $\log (\mathrm{M})=3$, corresponding to a chain with $10 \mathrm{MMA}$ units. Both lines together define the grey zone in Figure 11 . Using the log(M) values of 3 and 3.3 as an upper limit for biodegradability, it is clear that the majority of the MMA segments can be degraded by bacteria. The segments which are longer than 20 MMA units are mainly formed at lower overall monomer conversion during the synthesis. As the copolymerization has been carried out in batch the MMA unit incorporation is then very high and only decreasing at higher overall monomer conversions. In other words, the on target PMMA-like chains are only formed later on in the batch process. Reversly the very short chains are directly biodegradable as they are MDO-rich. As explained above, they are formed at the end of the radical copolymerization synthesis but they are not PMMA-like and therefore also off-spec. 
To obtain a PMMA-like product with an even beter degradability one should consider (i) either increasing the feed fraction of MDO, (ii) choose a comonomer combination with more suitable monomer reactivity ratios, preferably close to 1 , or (iii) synthesize the copolymer according to a semi-batch procedure. ${ }^{117}$ However, for the current purpose of highlighting the proof-of-concept of connecting synthesis and recycling of FRP-made copolymers a suboptimal set of reaction conditions featuring severe drifting is better suited to illustrate the general capability of the $k \mathrm{MC}$ tool. It should be further stressed that Figure 11 is also characterized by an excellent mathematical description of the tail of the distribution, as witnessed by the limited noise at the higher molar masses, highlighting the relevance of the compression-based matrix modeling framework in case high average chan length polymers are targeted.

\section{Conclusions}

Matrix-based $k M C$ modeling is a powerful tool to study both radical polymerization and degradation as it allows to store kinetic and molecular information on a chain-by-chain-basis so that specific reactions can be explicitly executed. Moreover, the introduced compression method enables to fully describe the shape of the log-MMD with a possible location of its tail in the high molar mass region, which is often characterized by elevated noise-to-signal ratios both based on experimental and more conventional computational analysis.

To illustrate the strength of matrix-baed $k M C$ modeling a differentiation has been made between homopolymers and copolymers considering MMA as the main monomer. For the former, a detailed isothermal lab-scale kinetic study of FRP of MMA and the subsequent thermal degradation with high monomer recovery has been performed. For the latter, focus has been on the understanding of the lab-scale isothermal kinetics of the radical copolymerization of MMA and MDO in view of controlling segment lengths to enable the transformation in biodegradable oligmers after hydrolysis in a later stage.

The log-MMD is a crucial molecular property to fully understand thermochemical recycling. During conventional synthesis this distribution can develop bimodality at higher monomer conversions, lower polymerization temperatures and lower initial conventional radical concentrations. This bimodality is caused by the prominent rol of the gel-effect and to fully grasp the detailed shape of the log-MMD one should additionally account for the glass and cage effect. During the thermal degradation the chains from the tail with weak head-to-head defects are first attacked. At higher polymer mass losses one also observes the contributions starting with fission at an unsaturated chain-end and head-to-tail fission, the latter supported by the presence of a high number of monomer units.

For the copolymerization of MMA and MDO under batch conditions the compositional drifting is strong so that inherently very relevant chain-to-chain deviations exist. This strong intermolecular copolymer hetereogeneity has a direct impact on the degradation behavior and it is an important insight for the chemical treatment of copolymers in general. This has been illustrated by showcasing that upon hydrolyis the copolymer chains that have been formed first during the copolymer synthesis possess too long MMA segments and those formed toward the end are characterized by too long MDO segments. In other words, only a specific fraction of copolymer chains has a regulated incorporation of the ester functional moieties. An inspection of the shift of the logMMD upon complete hydrolysis although reveals that the majority of the MMA segments has a length below a threshold value of 20 and even 10 in many situations.

Hence, it is clear that to study (thermal) degradation of (co)polymers it is beneficial to know in detail the composition of the starting polymer reactant. The developed $k M C$ strategy can be seen as an important tool to support the understanding of both polymerization, depolymerization and degradation kinetics and is applicable in the long run to design and connect both polymer synthesis and degradation at the level of the individual molecule. Future work will be aimed at widening the portfolio of polymer compositions addressed and the consideration of different operation modes, taking into account possible constraints due to scaleup and impurities.

\section{Conflicts of interest}

There are no conflicts to declare

\section{Acknowledgements}

K.D.S. acknowledges support by the Fund for Scientific Research Flanders (FWO Flanders) through a SBO-project (1SB0619N, 20192020). This work has also received funding from the European Union's Horizon 2020 research and innovation program under grant agreement No 820687. The Second Generation MethylMethAcrylate (MMAtwo) project related results presented reflect only the 
author's view. The Commission is not responsible for any use that may be made of the information it contains. P.H.M.V.S. acknowledges FWO Flanders for a postdoctoral fellowship. K.M.V.G. and D.R.D also acknowledge VLAIO/Catalisti regarding the WaTCH project.

\section{Notes and references}

1 P. Nesvadba, in Encyclopedia of Radicals in Chemistry, Biology and Materials, 2012.

2 J. M. Asua, Polymer Reaction Engineering, 2012.

3 S. M. Al-Salem, P. Lettieri and J. Baeyens, Waste Manag., 2009, 29, 2625-2643.

4 K. Ragaert, L. Delva and K. Van Geem, Waste Manag., 2017, 69, 24-58.

5 U. Ali, K. J. B. A. Karim and N. A. Buang, Polym. Rev., 2015, 55, 678-705.

6 E. K. C. Moens, K. De Smit, Y. W. Marien, A. D. Trigilio, P. H. M. Van Steenberge, K. M. Van Geem and D. R. D’ho oge, 1-49.

7 J. Scheirs and W. Kaminsky, Feedstock recycling and pyrolysis of waste plastics, 2006, vol. 2006.

8 D. S. Achilias, Eur. Polym. J., 2007, 43, 2564-2575.

9 W. Kaminsky and J. Franck, J. Anal. Appl. Pyrolysis, 1991, 19, 311-318.

10 W. Kaminsky and C. Eger, J. Anal. Appl. Pyrolysis, 2001, 58-59, 781-787.

11 T. Kashiwagi, J. E. Brown, A. Inaba, K. Hatada, T. Kitayama and E. Masuda, Macromolecules, 1986, 19, $2160-2168$.

12 T. Kashiwagi, T. Hirata and J. E. Brown, Macromolecules, 1985, 18, 131-138.

13 L. E. Manring, Macromolecules, 1987, 530, 528-530.

14 L. E. Manring, Macromolecules, 1991, 24, 3304-3309.

15 L. E. Manring, D. Y. Sogah and G. M. Cohen, Macromolecules, 1989, 22, 4652-4654.

16 T. Hirata, T. Kashiwagi and J. E. Brown, Macromolecules, 1985, 18, 1410-1418.

17 P. Nising, T. Zeilmann and T. Meyer, Chem. Eng. Technol., 2003, 26, 599-604.

18 K. Smolders and J. Baeyens, Waste Manag., 2004, 24, 849-857.

19 L. E. Manring, Macromolecules, 1989, 22, 2673-2677.

20 D. R. D'hooge, M. F. Reyniers and G. B. Marin, Macromol. React. Eng., 2013, 7, 362-379.

21 D. S. Achilias, Macromol. Theory Simulations, 2007, 16, 319-347.

22 M. Ferriol, A. Gentilhomme, M. Cochez, N. Oget and J. L. Mieloszynski, Polym. Degrad. Stab., 2003, 79, $271-281$.

23 Y. H. Hu and C. Y. Chen, Polym. Degrad. Stab., 2003, 82, 81-88.

24 A. Tardy, J. Honoré, J. Tran, I. Bataille, D. Letourneur, J. Perrier, C. Nicoletti, M. Maresca, D. Gigmes, J. Nicolas and Y. Guillaneuf, Angew. Chemie, , DOI:10.1002/anie.201707043.

25 A. Tardy, J. Nicolas, D. Gigmes, C. Lefay and Y. Guillaneuf, Chem. Rev., 2017, 117, 1319-1406.

26 D. Gigmes, P. H. M. Van Steenberge, D. Siri, D. R. D’hooge, Y. Guillaneuf and C. Lefay, Macromol. Rapid Commun., $2018,39,1-7$.

27 J. B. Lena and A. M. Van Herk, Ind. Eng. Chem. Res., 2019, 58, 20923-20931.

28 F. Kawai, Appl. Microbiol. Biotechnol., 1993, 39, 382-385.

29 D. R. D'Hooge, P. H. M. Van Steenberge, M. F. Reyniers and G. B. Marin, Prog. Polym. Sci., 2016, 58, 59-89.

30 S. K. Fierens, S. Telitel, P. H. M. Van Steenberge, M. F. Reyniers, G. B. Marin, J. F. Lutz and D. R. D'hooge, Macromolecules, 2016, 49, 9336-9344.

31 D. J. G. Devlaminck, P. H. M. Van Steenberge, L. De Keer, M.-F. Reyniers and D. R. D'hooge, Polym. Chem., 2017, 8, $6948-6963$.

32 P. H. M. Van Steenberge, B. Verbraeken, M. F. Reyniers, R. Hoogenboom and D. R. D'Hooge, Macromolecules, 2015, 48, 77657773.

33 A. Nasresfahani and R. A. Hutchinson, Ind. Eng. Chem. Res., 2018, 57, 9407-9419.

34 L. Wang and L. J. Broadbelt, Macromolecules, 2009, 42, 7961-7968.

35 P. Derboven, P. H. M. Van Steenberge, J. Vandenbergh, M. Reyniers, T. Junkers, R. D. D’hooge and G. B. Marin, Macromol. Rapid Commun., 2015, 2149-2155.

36 P. H. M. Van Steenberge and R. A. Hutchinson, AlChE J., 2019, 65, 1-13.

37 P. H. M. Van Steenberge, D. R. D'Hooge, M. F. Reyniers, G. B. Marin and M. F. Cunningham, Macromolecules, $2014,47,7732-7741$.

38 D. R. D'hooge, M.-F. Reyniers, F. J. Stadler, B. Dervaux, C. Bailly, F. E. Du Prez and G. B. Marin, Macromolecules, 2010, 43, 87668781.

39 S. K. Fierens, D. R. D’hooge, P. H. M. Van Steenberge, M. F. Reyniers and G. B. Marin, Polymers (Basel)., $2015,7,655-679$.

40 S. K. Fierens, P. H. M. Van Steenberge, M. F. Reyniers, G. B. Marin and D. R. D'hooge, AIChE J., 2017, 63, 4971-4986.

41 S. K. Fierens, P. H. M. Van Steenberge, F. Vermeire, M. F. Reyniers, G. B. Marin and D. R. D'hooge, AlChE J., $2018,64,2545-2559$.

42 D. R. D'hooge, M. F. Reyniers and G. B. Marin, Macromol. React. Eng., 2013, 7, 362-379.

43 D. R. D'hooge, M.-F. Reyniers and G. B. Marin, Macromol. React. Eng., 2009, 3, 185-209.

44 P. H. M. Van Steenberge, J. Vandenbergh, D. R. D'Hooge, M. F. Reyniers, P. J. Adriaensens, L. Lutsen, D. J. M. Vanderzande and G. B. Marin, Macromolecules, 2011, 44, 8716-8726.

45 I. Kryven and P. D. ledema, Macromol. Theory Simulations, 2013, 22, 89-106.

46 V. Schamboeck, I. Kryven and P. D. Iedema, Macromol. Theory Simulations, , DOI:10.1002/mats.201700047.

47 J. Bonilla, E. Saldívar, A. Flores-Tlacuahuac, E. Vivaldo-Lima, R. Pfaendner and F. Tiscareño-Lechuga, Polym. React. Eng., 2002, 10, 227-263.

48 N. De Rybel, P. H. M. Van Steenberge, M. F. Reyniers, D. R. D’hooge and G. B. Marin, Chem. Eng. Sci., 2018, 177, 163-179.

49 P. H. M. Van Steenberge, D. R. D'Hooge, J. Vandenbergh, M. F. Reyniers, P. J. Adriaensens, D. J. M. Vanderzande and G. B. Marin, Macromol. Theory Simulations, 2013, 22, 246-255.

50 P. H. M. Van Steenberge, D. R. D’Hooge, Y. Wang, M. Zhong, M. F. Reyniers, D. Konkolewicz, K. Matyjaszewski and G. B. Marin, Macromolecules, 2012, 45, 8519-8531.

51 N. De Rybel, P. H. M. Van Steenberge, M. F. Reyniers, C. Barner-Kowollik, D. R. D'hooge and G. B. Marin, Macromol. Theory Simulations, 2017, 26, 1-19. 
52 J. C. Hernández-Ortiz, P. H. M. Van Steenberge, J. N. E. Duchateau, C. Toloza, F. Schreurs, M.-F. Reyniers, G. B. Marin and D. R. D'Hooge, Chem. Eng. J., 2019, 377, 119980.

53 D. T. Gillespie, J. Phys. Chem., 1977, 81, 2340-2361.

54 P. Derboven, D. R. D’hooge, M.-F. Reyniers, G. B. Marin and C. Barner-Kowollik, Macromolecules, $2015,48,492-501$.

55 S. K. Fierens, D. R. D’hooge, P. H. M. Van Steenberge, M. F. Reyniers and G. B. Marin, Chem. Eng. J., 2015, 278, 407-420.

56 P. H. M. Van Steenberge, D. R. D’Hooge, Y. Wang, M. Zhong, M. F. Reyniers, D. Konkolewicz, K. Matyjaszewski and G. B. Marin, Macromolecules, 2012, 45, 8519-8531.

57 T. M. Kruse, O. Sang Woo and L. J. Broadbelt, Chem. Eng. Sci., 2001, 56, 971-979.

58 T. M. Kruse, O. S. Woo, H. W. Wong, S. S. Khan and L. J. Broadbelt, Macromolecules, 2002, 35, 7830-7844.

59 G. B. Desmet, Y. W. Marien, P. H. M. Van Steenberge, D. R. D'Hooge, M. F. Reyniers and G. B. Marin, Polym. Chem., 2017, 8, 71437150.

60 N. De Rybel, P. H. M. Van Steenberge, M. F. Reyniers, D. R. D’hooge and G. B. Marin, Macromolecules, $2019,52,4555-4569$.

61 O. Monyatsi, A. N. Nikitin and R. A. Hutchinson, Macromolecules, 2014, 47, 8145-8153.

62 V. C. Chappa, C. Pastorino, M. F. Del Grosso, C. R. Arbeitman, M. Müller and G. García Bermúdez, Nucl. Instruments Methods Phys. Res. Sect. B Beam Interact. with Mater. Atoms, 2010, 268, 3235-3238.

63 H. Galina and J. B. Lechowicz, Polymer (Guildf)., 2000, 41, 615-619.

64 G. Jahanmir, M. J. Abdekhodaie and Y. Chau, Macromolecules, 2018, 51, 3941-3952.

65 K. Sevim and J. Pan, Acta Biomater., 2018, 66, 192-199.

66 K. N. S. Adema, H. Makki, E. A. J. F. Peters, J. Laven, L. G. J. Van Der Ven, R. A. T. M. Van Benthem and G. De With, Phys. Chem. Chem. Phys., 2015, 17, 19962-19976.

67 P. D. ledema, K. Remerie, M. van der Ham, E. Biemond and J. Tacx, Chem. Eng. Sci., 2011, 66, 5474-5486.

68 R. Vinu, S. E. Levine, L. Wang and L. J. Broadbelt, Chem. Eng. Sci., 2012, 69, 456-471.

69 E. V. Bystritskaya, O. N. Karpukhin and A. V. Kutsenova, Int. J. Polym. Sci., , DOI:10.1155/2011/849370.

70 E. V. Bystritskaya, O. N. Karpukhin and A. V. Kutsenova, Russ. J. Phys. Chem. B, 2013, 7, 478-484.

71 S. M. Bose and Y. Git, Macromol. Theory Simulations, 2004, 13, 453-473.

72 C. Huang, C. Tzoganakis and T. A. Duever, Polym. React. Eng., 1995, 3, 43-63.

73 T. Radiotis and G. R. Brown, J. Macromol. Sci. - Pure Appl. Chem., 1997, 34, 743-757.

74 M. Guaita, O. Chiantore and M. P. Luda, Macromolecules, 1990, 23, 2087-2092.

75 M. Guaita, O. Chiantore and M. P. Luda, Macromolecules, 1991, 24, 2198-2202.

76 H. Tobita, Macromolecules, 1993, 836-841.

77 R. Giudici and A. E. Hamielec, Polym. React. Eng., 1996, 4, 73-101.

78 H. Tobita, Macromolecules, 1996, 29, 3010-3021.

79 H. Tobita, J. Polym. Sci. Part B Polym. Phys., 2001, 39, 391-403.

80 H. Tobita, Macromolecules, 1996, 29, 3000-3009.

81 D. S. Achilias and C. Kiparissides, Macromolecules, 1992, 25, 3739-3750.

82 G. Johnston-Hall, M. H. Stenzel, T. P. Davis, C. Barner-Kowollik and M. J. Monteiro, Macromolecules, 2007, 40, 2730-2736.

83 S. W. Prescott, Macromolecules, 2003, 36, 9608-9621.

84 M. Buback, B. Huckestein, F. -D Kuchta, G. T. Russell and E. Schmid, Macromol. Chem. Phys., 1994, 195, $2117-2140$.

85 D. R. D'Hooge, P. H. M. Van Steenberge, M. F. Reyniers and G. B. Marin, Polymers (Basel)., $2014,6,1074-1095$.

86 S. K. Fierens, R. D. Dagmar, P. H. M. Van Steenberge, M. Reyniers and G. B. Marin, Chem. Eng. J., , DOI:10.1016/j.cej.2014.09.024.

87 A. L. T. Brandão, J. B. P. Soares, J. C. Pinto and A. L. Alberton, Macromol. React. Eng., 2015, 9, 141-185.

88 K. A. Payne, D. R. D'Hooge, P. H. M. Van Steenberge, M. F. Reyniers, M. F. Cunningham, R. A. Hutchinson and G. B. Marin, Macromolecules, 2013, 46, 3828-3840.

89 D. Panke, Macromol. Theory Simulations, 1995, 4, 759-772.

90 D. Panke, M. Stickler and W. Wunderlich, Die Makromol. Chemie, 1983, 184, 175-191.

91 G. Moad, Prog. Polym. Sci., 2019, 88, 130-188.

92 D. F. Sangster, J. Feldthusen, J. Strauch and C. M. Fellows, Macromol. Chem. Phys., 2008, 209, 1612-1627.

93 J. M. Asua, S. Beuermann, M. Buback, P. Castignolles, B. Charleux, R. G. Gilbert, R. A. Hutchinson, J. R. Leiza, A. N. Nikiti n, J. P. Vairon and A. M. Van Herk, Macromol. Chem. Phys., 2004, 205, 2151-2160.

94 S. Beuermann and M. Buback, Prog. Polym. Sci., 2002, 27, 191-254.

95 S. Beuermann, M. Buback, T. P. Davis, R. G. Gilbert, R. A. Hutchinson, O. F. Olaj, G. T. Russell, J. Schweer and A. M. Van He rk, Macromol. Chem. Phys., 1997, 198, 1545-1560.

96 C. Barner-Kowollik, S. Beuermann, M. Buback, P. Castignolles, B. Charleux, M. L. Coote, R. A. Hutchinson, T. Junkers, I. Lacík, G. T. Russell, M. Stach and A. M. Van Herk, Polym. Chem., 2014, 5, 204-212.

97 G. Johnston-Hall and M. J. Monteiro, Prog. Polym. Sci., 2008, 46, 936-946.

98 G. Johnston-Hall and M. J. Monteiro, Macromolecules, 2007, 40, 7171-7179.

99 G. Johnston-Hall, A. Theis, M. J. Monteiro, T. P. Davis, M. H. Stenzel and C. Barner-Kowollik, Macromol. Chem. Phys., 2005, 206, 2047-2053.

100 M. Stickler and G. Meyerhoff, Die Makromol. Chemie, 1978, 179, 2729-2745.

101 D. Meimaroglou and C. Kiparissides, Ind. Eng. Chem. Res., 2014, 53, 8963-8979.

102 Y. W. Marien, P. H. M. Van Steenberge, C. Barner-Kowollik, M. F. Reyniers, G. B. Marin and D. R. D'Hooge, Macromolecules, 2017, 50, 1371-1385.

103 Y. Nakamura and S. Yamago, Macromolecules, 2015, 48, 6450-6456.

104 D. Achilias and C. Kiparissides, J. Appl. Polym. Sci., 1988, 35, 1303-1323.

105 V. E. Trommsdorff, H. Köhle and P. Lagally, Die Makromol. Chemie, 1948, 1, 169-198.

106 C. Barner-Kowollik and G. T. Russell, Prog. Polym. Sci., 2009, 34, 1211-1259.

107 F. L. Marten and A. E. Hamielec, in ACS Symposium Series, 1979, pp. 43-70.

108 T. Kashiwagi, A. Inabi and A. Hamins, Polym. Degrad. Stab., 1989, 26, 161-184.

109 S. Agarwal, Polym. J., 2007, 39, 163-174. 
110 S. T. Balke and A. E. Hamielec, J. Appl. Polym. Sci., 1973, 17, 905-949.

111 L. De Keer, M.-F. Van Steenberge, Paul H.M. Reyniers, L. Seda, K.-D. Hungenberg, D. R. D’hooge and G. B. Marin, AlChE J., 2012, 59, 215-228.

112 P. Derboven, P. H. M. Van Steenberge, M. F. Reyniers, C. Barner-Kowollik, D. R. D'hooge and G. B. Marin, Macromol. Theory Simulations, 2016, 25, 104-115.

113 F. R. Mayo and F. M. Lewis, J. Am. Chem. Soc., 1944, 66, 1594-1601.

114 T. R. Rooney, A. Chovancová, I. Lacík and R. A. Hutchinson, Polymer (Guildf)., 2017, 130, 39-49.

115 M. L. Coote, M. D. Zammit, T. P. Davis and G. D. Willett, Macromolecules, 1997, 30, 8182-8190.

116 N. Kazemi, T. A. Duever and A. Penlidis, Macromol. React. Eng., 2011, 5, 385-403.

117 J. Lena, A. W. Jackson, L. R. Chennamaneni, C. T. Wong, F. Lim, Y. Andriani, P. Thoniyot and A. M. Van Herk, Macromolecules, DOI:10.1021/acs.macromol.0c00207.

118 M. Al-Harthi, M. J. Khan, S. H. Abbasi and J. B. P. Soares, Macromol. React. Eng., 2009, 3, 148-159. 